Relativistic Hartree-Fock calculations of transition rates for allowed and forbidden lines in $\mathrm{Nd}$ IV

This content has been downloaded from IOPscience. Please scroll down to see the full text. 2014 J. Phys. B: At. Mol. Opt. Phys. 47035002

(http://iopscience.iop.org/0953-4075/47/3/035002)

View the table of contents for this issue, or go to the journal homepage for more

Download details:

IP Address: 35.46.1.85

This content was downloaded on 13/01/2014 at 08:37

Please note that terms and conditions apply. 


\title{
Relativistic Hartree-Fock calculations of transition rates for allowed and forbidden lines in Nd IV
}

\author{
S Enzonga Yoca ${ }^{1}$ and P Quinet ${ }^{2,3}$ \\ ${ }^{1}$ Faculté des Sciences et Techniques, Département de Physique, Université Marien Ngouabi, \\ BP 69 Brazzaville, Congo \\ ${ }^{2}$ Astrophysique et Spectroscopie, Université de Mons - UMONS, B-7000 Mons, Belgium \\ ${ }^{3}$ IPNAS, Université de Liège, B-4000 Liège, Belgium \\ E-mail: quinet@umons.ac.be
}

Received 22 November 2013

Accepted for publication 11 December 2013

Published 10 January 2014

\begin{abstract}
A pseudo-relativistic Hartree-Fock model including a large amount of configurationinteraction effects has been used to compute radiative decay rates for allowed and forbidden transitions in Nd IV. Detailed comparisons of transition probabilities, oscillator strengths and radiative lifetimes with data previously published are also reported and discussed in the present work.
\end{abstract}

Keywords: atomic data, transition rates, triply ionized lanthanides

\section{Introduction}

It is well known that triply ionized lanthanides play an important role in different areas such as laser physics, lighting industry, photonics, molecular biology, medical diagnostics, etc (see e.g. Hemmilä 1995, Wybourne 2004, Hasegawa et al 2004, Dossing 2005) and their radiative properties were essentially investigated from spectroscopy experiments on ions embedded in compounds or crystal lattices.

In the case of neodymium $(Z=60)$, the emission spectrum of $\mathrm{Nd}^{3+}$ ion has been studied in various compounds such as, for example, in $\mathrm{NaCl}$ and $\mathrm{KCl}$ single crystals (Rao 1973), in yttrium orthovanadate (Wortman et al 1974), in neodymium trifluorides (Vaishnava et al 1974), in $\mathrm{Nd}^{3+}: \mathrm{Y}_{3} \mathrm{Al}_{5} \mathrm{O}_{12}$ (Gasparik and Ozvoldova 1974), in $\mathrm{LaCl}_{3}: \mathrm{Nd}^{3+}$ (Zalucha et al 1974 , Crosswhite et al 1976) or in $\mathrm{Gd}_{2}\left(\mathrm{MoO}_{4}\right)_{3}$ (Spector et al 1977).

The free $\mathrm{Nd}^{3+}$ ion spectrum was first observed by Irwin (1968) who listed about 1000 of the strongest $\mathrm{Nd}$ lines obtained using a spark source in the region 127-311 nm. A number of lines were classified as $4 \mathrm{f}^{3}{ }^{4} \mathrm{I}^{\circ}-4 \mathrm{f}^{2} 5 \mathrm{~d}, 4 \mathrm{f}^{2}\left({ }^{3} \mathrm{H}\right) 6 \mathrm{~s}-$ $4 \mathrm{f}^{2}\left({ }^{3} \mathrm{H}\right) 6 \mathrm{p}$, or $4 \mathrm{f}^{2}\left({ }^{3} \mathrm{H}\right) 5 \mathrm{~d}-4 \mathrm{f}^{2}\left({ }^{3} \mathrm{H}\right) 6 \mathrm{p}$ transitions in $\mathrm{Nd} \mathrm{IV}$, but no connection between any of the three resulting systems was found. Consequently, this study was not considered as reliable enough to be included in the critical compilation by Martin et al
(1978) who preferred to list the levels of the $4 \mathrm{f}^{3}$ configuration taken from the analysis of the $\mathrm{Nd}^{3+}$ spectrum in a $\mathrm{LaCl}_{3}$ host crystal due to Crosswhite et al (1976).

More recently, the first level structure investigation of the free $\mathrm{Nd}^{3+}$ ion was reported by Wyart et al (2006) who observed the neodymium emission spectrum produced by vacuum spark sources in the vacuum ultraviolet on two normal-incidence spectrographs. They identified more than 550 lines as transitions from $854 \mathrm{f}^{2} 5 \mathrm{~d}$ levels to the lowest 37 levels of the $4 \mathrm{f}^{3}$ ground configuration. Shortly after this work, the same experimental material was used by Wyart et al (2007) to extend wavelength measurements in higher excitation conditions. This led to the completion to all 41 levels of the $4 \mathrm{f}^{3}$ ground configuration and to the classification of 1426 lines involving the excited configurations $4 f^{2} 5 d, 4 f^{2} 6 p$, $4 \mathrm{f}^{2} 6 \mathrm{~s}$. A number of new levels were also assigned to the coreexcited $5 \mathrm{p}^{5} 4 \mathrm{f}^{4}$ configuration. Altogether 111 odd parity and 121 even parity levels were established. In this latter work, theoretical values of lifetimes and transition probabilities, computed with a rather limited relativistic Hartree-Fock (HFR) model, were reported. Furthermore, Wyart et al (2008) carried out a detailed theoretical investigation of the $4 \mathrm{f}^{3}$ configuration in Nd IV by performing a parametric fit of level 
Table 1. Adopted radial parameters $\left(\mathrm{in}^{-1}\right.$ ) for even-parity configurations of Nd IV.

\begin{tabular}{|c|c|c|c|c|c|}
\hline Configuration & Parameter & HFR & Fitted & $\begin{array}{l}\text { Fitted/ } \\
\text { HFR }\end{array}$ & Note $^{\mathrm{a}}$ \\
\hline $4 f^{3}$ & $\begin{array}{l}\mathrm{E}_{\mathrm{av}} \\
\mathrm{F}^{2}(4 \mathrm{f}, 4 \mathrm{f}) \\
\mathrm{F}^{4}(4 \mathrm{f}, 4 \mathrm{f}) \\
\mathrm{F}^{6}(4 \mathrm{f}, 4 \mathrm{f}) \\
\alpha \\
\beta \\
\gamma \\
\zeta_{4 \mathrm{f}}\end{array}$ & $\begin{array}{ll}- & \\
102658 \\
64418 \\
46345 \\
- \\
- \\
-\quad \\
- & 957\end{array}$ & $\begin{array}{r}25954 \\
79427 \\
55720 \\
38123 \\
10 \\
-324 \\
878 \\
890\end{array}$ & $\begin{array}{l}- \\
0.77 \\
0.86 \\
0.82 \\
- \\
- \\
- \\
0.93\end{array}$ & \\
\hline $4 f^{2} 6 p$ & $\begin{array}{l}\mathrm{E}_{\mathrm{av}} \\
\mathrm{F}^{2}(4 \mathrm{f}, 4 \mathrm{f}) \\
\mathrm{F}^{4}(4 \mathrm{f}, 4 \mathrm{f}) \\
\mathrm{F}^{6}(4 \mathrm{f}, 4 \mathrm{f}) \\
\alpha \\
\beta \\
\gamma \\
\zeta_{4 \mathrm{f}} \\
\zeta_{6 \mathrm{p}} \\
\mathrm{F}^{2}(4 \mathrm{f}, 6 \mathrm{p}) \\
\mathrm{G}^{2}(4 \mathrm{f}, 6 \mathrm{p}) \\
\mathrm{G}^{4}(4 \mathrm{f}, 6 \mathrm{p})\end{array}$ & $\begin{array}{rr}- & \\
110783 \\
69929 \\
50430 \\
- \\
- \\
- \\
\\
\\
1059 \\
2962 \\
9951 \\
2518 \\
2313\end{array}$ & $\begin{array}{r}164287 \\
82525 \\
62129 \\
39513 \\
19 \\
-481 \\
1569 \\
986 \\
3589 \\
7762 \\
2374 \\
2011\end{array}$ & $\begin{array}{l} \\
0.93 \\
1.21 \\
0.78 \\
0.94 \\
0.87\end{array}$ & $\begin{array}{l}\mathrm{r} 1 \\
\mathrm{r} 1 \\
\mathrm{r} 1\end{array}$ \\
\hline $5 p^{5} 4 f^{4}$ & $\begin{array}{l}\mathrm{E}_{\mathrm{av}} \\
\mathrm{F}^{2}(4 \mathrm{f}, 4 \mathrm{f}) \\
\mathrm{F}^{4}(4 \mathrm{f}, 4 \mathrm{f}) \\
\mathrm{F}^{6}(4 \mathrm{f}, 4 \mathrm{f}) \\
\alpha \\
\beta \\
\gamma \\
\zeta_{4 \mathrm{f}} \\
\zeta_{5 \mathrm{p}} \\
\mathrm{F}^{2}(4 \mathrm{f}, 5 \mathrm{p}) \\
\mathrm{G}^{2}(4 \mathrm{f}, 5 \mathrm{p}) \\
\mathrm{G}^{4}(4 \mathrm{f}, 5 \mathrm{p})\end{array}$ & $\begin{array}{rr}- & \\
& 96503 \\
60281 \\
43289 \\
- \\
- \\
-\end{array}$ & $\begin{array}{r}183596 \\
73412 \\
53344 \\
34742 \\
20 \\
-496 \\
1616 \\
793 \\
16522 \\
37082 \\
25046 \\
15532\end{array}$ & $\begin{array}{l} \\
0.90 \\
1.02 \\
0.73 \\
0.86 \\
0.71\end{array}$ & $\begin{array}{l}\mathrm{r} 2 \\
\mathrm{r} 2 \\
\mathrm{r} 2\end{array}$ \\
\hline $4 f^{3}-4 f^{2} 6 p$ & $\begin{array}{l}R^{2}(4 f 4 f, 4 f 6 p) \\
R^{4}(4 f 4 f, 4 f 6 p)\end{array}$ & $\begin{array}{l}-4973 \\
-3193\end{array}$ & $\begin{array}{l}-4227 \\
-2714\end{array}$ & $\begin{array}{l}0.85 \\
0.85\end{array}$ & $\begin{array}{l}\mathrm{f} \\
\mathrm{f}\end{array}$ \\
\hline $4 f^{3}-5 p^{5} 4 f^{4}$ & $\begin{array}{l}\mathrm{R}^{2}(4 \mathrm{f} 5 \mathrm{p}, 4 \mathrm{f} 4 \mathrm{f}) \\
\mathrm{R}^{4}(4 \mathrm{f} 5 \mathrm{p}, 4 \mathrm{f} 4 \mathrm{f}) \\
\mathrm{R}^{2}(5 \mathrm{p} 5 \mathrm{p}, 4 \mathrm{f} 5 \mathrm{p})\end{array}$ & $\begin{array}{l}-21791 \\
-12092 \\
-39045\end{array}$ & $\begin{array}{l}-18539 \\
-10288 \\
-33218\end{array}$ & $\begin{array}{l}0.85 \\
0.85 \\
0.85\end{array}$ & $\begin{array}{l}\text { r3 } \\
\text { r3 } \\
\text { r3 }\end{array}$ \\
\hline
\end{tabular}

a $r$ stands for 'parameters varied in a constant ratio' and $f$ stands for 'fixed parameter value'.

energies, taking into account Coulomb and spin-dependent interactions beyond the first order of perturbation. Note also that systematic Hartree-Fock and relativistic configurationinteraction studies of energy levels and lifetimes of some triply ionized lanthanides, including $\mathrm{Nd}^{3+}$, were published by Dzuba et al (2003).

In the present work, the relativistic Hartree-Fock method including a large amount of configuration interaction has been used to compute the radiative parameters corresponding to allowed and forbidden transitions in Nd IV. These calculations are an extension of our previous studies of triply ionized lanthanides La IV (Biémont et al 2009), Ce IV (Zhang et al 2001a), Pr IV (Enzonga Yoca and Quinet 2013) and Yb IV (Wyart et al 2001).
Table 2. Adopted radial parameters $\left(\mathrm{in}^{-1}\right.$ ) for even-parity configurations of Nd IV.

\begin{tabular}{|c|c|c|c|c|c|}
\hline Configuration & Parameter & HFR & Fitted & $\begin{array}{l}\text { Fitted/ } \\
\text { HFR }\end{array}$ & Note $^{\mathrm{a}}$ \\
\hline \multirow{14}{*}{$4 f^{2} 5 d$} & $\mathrm{E}_{\mathrm{g}}$ & _ & 90848 & _- & \\
\hline & $F^{2}(4 f, 4 f)$ & 109950 & 85551 & 0.78 & \\
\hline & $F^{4}(4 f, 4 f)$ & 69359 & 62158 & 0.90 & \\
\hline & $F^{6}(4 f, 4 f)$ & 50007 & 42335 & 0.85 & \\
\hline & $\alpha$ & - & 13 & - & \\
\hline & $\beta$ & - & -371 & - & \\
\hline & $\gamma$ & - & 822 & - & \\
\hline & $\zeta_{4 \mathrm{f}}$ & 1052 & 1004 & 0.95 & \\
\hline & $\zeta_{5 \mathrm{~d}}$ & 1146 & 1116 & 0.97 & \\
\hline & $\mathrm{F}^{2}(4 \mathrm{f}, 5 \mathrm{~d})$ & 30830 & 24269 & 0.79 & \\
\hline & $\mathrm{F}^{4}(4 \mathrm{f}, 5 \mathrm{~d})$ & 15288 & 14473 & 0.95 & \\
\hline & $\mathrm{G}^{1}(4 \mathrm{f}, 5 \mathrm{~d})$ & 13343 & 11749 & 0.88 & \\
\hline & $G^{3}(4 f, 5 d)$ & 11335 & 9718 & 0.86 & \\
\hline & $G^{5}(4 f, 5 d)$ & 8788 & 8099 & 0.92 & \\
\hline \multirow[t]{9}{*}{$4 f^{2} 6 s$} & $\mathrm{E}_{\mathrm{av}}$ & - & 123089 & - & \\
\hline & $F^{2}(4 f, 4 f)$ & 110707 & 83730 & 0.76 & \\
\hline & $F^{4}(4 f, 4 f)$ & 69878 & 61732 & 0.88 & \\
\hline & $F^{6}(4 f, 4 f)$ & 50392 & 42942 & 0.85 & \\
\hline & $\alpha$ & - & 14 & & $\mathrm{r} 1$ \\
\hline & $\beta$ & - & -379 & & $\mathrm{r} 1$ \\
\hline & $\gamma$ & - & 839 & & $\mathrm{r} 1$ \\
\hline & $\zeta_{4 \mathrm{f}}$ & 1059 & 994 & 0.94 & \\
\hline & $\mathrm{G}^{3}(4 \mathrm{f}, 6 \mathrm{~s})$ & 3365 & 2875 & 0.85 & \\
\hline \multirow[t]{2}{*}{$4 f^{2} 5 d-4 f^{2} 6 s$} & $R^{2}(4 f 5 d, 4 f 6 s)$ & 974 & 828 & 0.85 & $\mathrm{f}$ \\
\hline & $\mathrm{R}^{3}(4 \mathrm{f} 5 \mathrm{~d}, 4 \mathrm{f} 6 \mathrm{~s})$ & 2962 & 2518 & 0.85 & $\mathrm{f}$ \\
\hline
\end{tabular}

\section{Relativistic Hartree-Fock calculations}

To correctly describe the structure of a heavy atomic system such as triply ionized neodymium, it is necessary to consider the most important electronic correlation and relativistic effects simultaneously. We have already shown in many previous works on lowly charged lanthanide atoms that the pseudo-relativistic Hartree-Fock method of Cowan (1981) appears as a suitable compromise between a gratifying accuracy of the results (tested by comparison with accurate laser lifetime measurements), the moderate complexity of the codes used and the ability to obtain a large number of new results in a limited CPU time (see e.g. Quinet et al 2002, Biémont and Quinet 2003).

$\mathrm{Nd}^{3+}$ ion belongs to the $\mathrm{La}$ isoelectronic sequence in which two ions, i.e. $\mathrm{Ce}^{+}$and $\operatorname{Pr}^{2+}$ have already been studied with the same HFR approach (see Palmeri et al 2000a, 2000b, Biémont et al 2001, Zhang et al 2001b). In those works, $\mathrm{Ce}^{+}$and $\operatorname{Pr}^{2+}$ were considered as atomic systems with three valence electrons surrounding a Xe-like ionic core so that most of the intravalence correlation was represented within a configuration-interaction scheme, while core-valence correlation was described by a corepolarization model potential depending on two parameters, i.e. the static dipole polarizability of the ionic core, $\alpha_{d}$, and the cut-off radius, $r_{c}$. This picture is no more valid in the case of $\mathrm{Nd}^{3+}$ due to the fact that some core-excited configurations with an open $5 \mathrm{p}$ subshell are expected to 
Table 3. Computed oscillator strengths and transition probabilities for electric dipole lines in Nd IV. Only transitions with log $g f \geqslant-1.0$ are listed in the table.

\begin{tabular}{|c|c|c|c|c|c|c|c|c|}
\hline \multirow[b]{2}{*}{ Wavelength $^{\mathrm{a}}(\mathrm{nm})$} & \multicolumn{3}{|c|}{ Lower level $^{\mathrm{b}}$} & \multicolumn{3}{|c|}{ Upper level $^{\mathrm{b}}$} & \multirow[b]{2}{*}{$\log g f^{\mathrm{c}}$} & \multirow[b]{2}{*}{$g A^{\mathrm{c}}\left(\mathrm{s}^{-1}\right)$} \\
\hline & $\mathrm{E}\left(\mathrm{cm}^{-1}\right)$ & $J$ & Parity & $\mathrm{E}\left(\mathrm{cm}^{-1}\right)$ & $J$ & Parity & & \\
\hline $106.8300 *$ & 86678.25 & 7.5 & (e) & 180284.95 & 7.5 & (o) & -0.83 & $8.72 \mathrm{E}+08$ \\
\hline 119.2535 & 81303.91 & 6.5 & (e) & 165158.58 & 5.5 & (o) & -0.92 & $5.65 \mathrm{E}+08$ \\
\hline 119.4932 & 77809.61 & 4.5 & (e) & 161496.05 & 3.5 & (o) & -0.75 & $8.37 \mathrm{E}+08$ \\
\hline 119.6176 & 75666.91 & 3.5 & (e) & 159266.55 & 2.5 & (o) & -1.00 & $4.69 \mathrm{E}+08$ \\
\hline 120.0294 & 77912.50 & 5.5 & (e) & 161225.30 & 4.5 & (o) & -0.85 & $6.51 E+08$ \\
\hline 120.8150D & 73366.17 & 4.5 & (e) & 156137.08 & 4.5 & (o) & -0.92 & $5.55 \mathrm{E}+08$ \\
\hline 121.3802 & 79189.02 & 6.5 & (e) & 161574.84 & 5.5 & (o) & -0.71 & $8.83 \mathrm{E}+08$ \\
\hline $121.4453 *$ & 71744.57 & 4.5 & (e) & 154086.17 & 5.5 & (o) & -0.67 & $9.75 \mathrm{E}+08$ \\
\hline 122.0447 & 98437.09 & 6.5 & (e) & 180284.95 & 7.5 & (o) & -0.69 & $9.08 \mathrm{E}+08$ \\
\hline 122.0632 & 77912.50 & 5.5 & (e) & 159837.56 & 4.5 & (o) & -0.54 & $1.28 \mathrm{E}+09$ \\
\hline 122.1247 & 71744.57 & 5.5 & (e) & 153628.30 & 4.5 & (o) & -0.64 & $1.03 \mathrm{E}+09$ \\
\hline 122.3860 & 84092.98 & 6.5 & (e) & 165802.12 & 5.5 & (o) & -0.38 & $1.84 \mathrm{E}+09$ \\
\hline $122.6015 \mathrm{D}$ & 74571.83 & 5.5 & (e) & 156137.08 & 4.5 & (o) & -0.56 & $1.21 \mathrm{E}+09$ \\
\hline $122.6582 *$ & 74673.95 & 6.5 & (e) & 156201.33 & 6.5 & (o) & -0.84 & $6.36 \mathrm{E}+08$ \\
\hline $122.7806^{*}$ & 73366.17 & 4.5 & (e) & 154812.25 & 3.5 & (o) & -0.86 & $6.10 \mathrm{E}+08$ \\
\hline 123.2369 & 80080.41 & 5.5 & (e) & 161225.30 & 4.5 & (o) & -0.77 & $7.47 \mathrm{E}+08$ \\
\hline $123.9323 \mathrm{D}$ & 74673.95 & 6.5 & (e) & 155363.06 & 5.5 & (o) & -0.68 & $9.20 \mathrm{E}+08$ \\
\hline 124.1824 & 81375.35 & 3.5 & (e) & 161901.81 & 2.5 & (o) & -0.98 & $4.49 \mathrm{E}+08$ \\
\hline 124.5693 & 81375.35 & 3.5 & (e) & 161652.20 & 3.5 & (o) & -0.81 & $6.67 \mathrm{E}+08$ \\
\hline 124.5919 & 73366.17 & 4.5 & (e) & 153628.30 & 4.5 & (o) & -0.55 & $1.21 \mathrm{E}+09$ \\
\hline 124.6625 & 74363.99 & 3.5 & (e) & 154581.00 & 2.5 & (o) & -0.76 & $7.46 \mathrm{E}+08$ \\
\hline 124.9920 & 78864.24 & 7.5 & (e) & 158869.30 & 6.5 & (o) & -0.49 & $1.38 \mathrm{E}+09$ \\
\hline 125.1713 & 79694.96 & 4.5 & (e) & 159585.52 & 3.5 & (o) & -0.69 & $8.75 \mathrm{E}+08$ \\
\hline 125.1826 & 81691.09 & 5.5 & (e) & 161574.84 & 5.5 & (o) & -0.79 & $6.96 \mathrm{E}+08$ \\
\hline 125.3300 & 78864.24 & 7.5 & (e) & 158653.53 & 7.5 & (o) & -0.03 & $3.96 \mathrm{E}+09$ \\
\hline 125.3806 & 80080.41 & 5.5 & (e) & 159837.56 & 4.5 & (o) & -0.61 & $1.04 \mathrm{E}+09$ \\
\hline 125.4213 & 80080.41 & 5.5 & (e) & 159812.28 & 5.5 & (o) & -0.47 & $1.44 \mathrm{E}+09$ \\
\hline 125.4235 & 76471.40 & 6.5 & (e) & 156201.33 & 6.5 & (o) & -0.34 & $1.93 E+09$ \\
\hline 125.5462 & 73366.17 & 4.5 & (e) & 153018.22 & 3.5 & (o) & -0.22 & $2.57 \mathrm{E}+09$ \\
\hline 125.7324 & 81691.09 & 5.5 & (e) & 161225.30 & 4.5 & (o) & -0.60 & $1.06 \mathrm{E}+09$ \\
\hline 125.7635 & 74571.83 & 5.5 & (e) & 154086.17 & 5.5 & (o) & -0.25 & $2.39 \mathrm{E}+09$ \\
\hline 125.8668 & 100835.39 & 7.5 & (e) & 180284.95 & 7.5 & (o) & 0.04 & $4.62 \mathrm{E}+09$ \\
\hline 125.9253 & 74673.95 & 6.5 & (e) & 154086.17 & 5.5 & (o) & -0.17 & $2.85 \mathrm{E}+09$ \\
\hline 125.9461 & 76471.40 & 6.5 & (e) & 155870.45 & 5.5 & (o) & -0.56 & $1.15 \mathrm{E}+09$ \\
\hline 126.2202D & 73366.17 & 4.5 & (e) & 152592.92 & 4.5 & (o) & -0.40 & $1.68 \mathrm{E}+09$ \\
\hline 126.3498 & 75666.91 & 3.5 & (e) & 154812.25 & 3.5 & (o) & -0.72 & $8.02 \mathrm{E}+08$ \\
\hline 126.3967D & 86067.88 & 3.5 & (e) & 165183.48 & 2.5 & (o) & -0.96 & $4.57 \mathrm{E}+08$ \\
\hline 126.4317 & 73616.22 & 2.5 & (e) & 152710.30 & 2.5 & (o) & -0.38 & $1.73 \mathrm{E}+09$ \\
\hline 126.4916 & 74571.83 & 5.5 & (e) & 153628.30 & 4.5 & (o) & -0.38 & $1.75 \mathrm{E}+09$ \\
\hline $126.7561 *$ & 76471.40 & 6.5 & (e) & 155363.06 & 5.5 & (o) & -0.96 & $4.63 \mathrm{E}+08$ \\
\hline 126.8857 & 88097.78 & 5.5 & (e) & 166908.81 & 4.5 & (o) & -0.94 & 4.77E+08 \\
\hline 126.9077 & 87114.16 & 2.5 & (e) & 165911.59 & 3.5 & (o) & -0.77 & $6.94 \mathrm{E}+08$ \\
\hline 126.9217 & 80080.41 & 5.5 & (e) & 158869.30 & 6.5 & (o) & -0.50 & $1.30 \mathrm{E}+09$ \\
\hline 127.0048 & 87114.16 & 2.5 & (e) & 165851.25 & 3.5 & (o) & -0.67 & $8.81 \mathrm{E}+08$ \\
\hline $127.0172 \mathrm{D}$ & 81691.09 & 5.5 & (e) & 160420.64 & 4.5 & (o) & -0.15 & $2.92 \mathrm{E}+09$ \\
\hline $127.0172 \mathrm{D}$ & 87072.95 & 5.5 & (e) & 165802.12 & 5.5 & (o) & -0.51 & $1.29 \mathrm{E}+09$ \\
\hline 127.1108 & 20005.22 & 6.5 & (o) & 98676.42 & 5.5 & (e) & -0.82 & $6.25 \mathrm{E}+08$ \\
\hline 127.1264 & 77539.71 & 7.5 & (e) & 156201.33 & 6.5 & (o) & 0.19 & $6.45 \mathrm{E}+09$ \\
\hline 127.2300 & 80128.30 & 2.5 & (e) & 158725.98 & 1.5 & (o) & -0.99 & $4.24 \mathrm{E}+08$ \\
\hline 127.3750 & 81303.91 & 6.5 & (e) & 159812.28 & 5.5 & (o) & -0.49 & $1.32 \mathrm{E}+09$ \\
\hline 127.5016 & 81155.44 & 4.5 & (e) & 159585.52 & 3.5 & (o) & -0.58 & $1.09 \mathrm{E}+09$ \\
\hline $127.6453 \mathrm{D}$ & 100976.62 & 3.5 & (e) & 179319.09 & 2.5 & (o) & -0.35 & $1.82 \mathrm{E}+09$ \\
\hline 127.6943D & 101159.67 & 6.5 & (e) & 79471.41 & 5.5 & (o) & -0.30 & $2.06 \mathrm{E}+09$ \\
\hline $127.7694 \mathrm{~T}$ & 83309.16 & 6.5 & (e) & 161574.84 & 5.5 & (o) & -0.56 & $1.13 \mathrm{E}+09$ \\
\hline 127.8412 & 80431.49 & 8.5 & (e) & 158653.53 & 7.5 & (o) & 0.41 & $1.06 \mathrm{E}+10$ \\
\hline 127.8787 & 77809.61 & 4.5 & (e) & 156008.48 & 4.5 & (o) & -0.22 & $2.44 \mathrm{E}+09$ \\
\hline 128.0334 & 5988.51 & 7.5 & (o) & 84092.98 & 6.5 & (e) & -0.82 & $6.15 \mathrm{E}+08$ \\
\hline 128.1046 & 77809.61 & 4.5 & (e) & 155870.45 & 5.5 & (o) & -0.85 & $5.82 \mathrm{E}+08$ \\
\hline 128.1695D & 74571.83 & 5.5 & (e) & 152592.92 & 4.5 & (o) & -0.22 & $2.43 E+09$ \\
\hline 128.1963 & 81832.25 & 3.5 & (e) & 159837.56 & 4.5 & (o) & -0.82 & $6.18 \mathrm{E}+08$ \\
\hline 128.2737 & 77912.50 & 5.5 & (e) & 155870.45 & 5.5 & (o) & -0.77 & $6.90 \mathrm{E}+08$ \\
\hline 128.2836 & 80080.41 & 5.5 & (e) & 158032.30 & 5.5 & (o) & -0.16 & $2.84 \mathrm{E}+09$ \\
\hline 128.3841 & 81375.35 & 3.5 & (e) & 159266.55 & 2.5 & (o) & -0.86 & $5.56 \mathrm{E}+08$ \\
\hline
\end{tabular}


Table 3. (Continued.)

\begin{tabular}{|c|c|c|c|c|c|c|c|c|}
\hline \multirow[b]{2}{*}{ Wavelength $^{\mathrm{a}}(\mathrm{nm})$} & \multicolumn{3}{|c|}{ Lower level $^{\mathrm{b}}$} & \multicolumn{3}{|c|}{ Upper level $^{\mathrm{b}}$} & \multirow[b]{2}{*}{$\log g f^{\mathrm{c}}$} & \multirow[b]{2}{*}{$g A^{\mathrm{c}}\left(\mathrm{s}^{-1}\right)$} \\
\hline & $\mathrm{E}\left(\mathrm{cm}^{-1}\right)$ & $J$ & Parity & $\mathrm{E}\left(\mathrm{cm}^{-1}\right)$ & $J$ & Parity & & \\
\hline 128.4802 & 0.00 & 4.5 & (o) & 77833.03 & 3.5 & (e) & -0.78 & $6.62 \mathrm{E}+08$ \\
\hline 128.5379 & 1897.11 & 5.5 & (o) & 79694.96 & 4.5 & (e) & -0.73 & $7.55 \mathrm{E}+08$ \\
\hline 128.5609 & 3907.43 & 6.5 & (o) & 81691.09 & 5.5 & (e) & -0.71 & $7.78 \mathrm{E}+08$ \\
\hline 128.6924 & 88097.78 & 5.5 & (e) & 165802.12 & 5.5 & (o) & -0.45 & $1.42 \mathrm{E}+09$ \\
\hline 128.7463 & 83768.34 & 5.5 & (e) & 161574.84 & 5.5 & (o) & -0.36 & $1.77 \mathrm{E}+09$ \\
\hline 128.7719 & 88097.78 & 5.5 & (e) & 165754.41 & 4.5 & (o) & -0.06 & $3.48 \mathrm{E}+09$ \\
\hline 128.8407 & 76471.40 & 6.5 & (e) & 154086.17 & 5.5 & (o) & -0.44 & $1.46 \mathrm{E}+09$ \\
\hline 128.8570 & 79694.96 & 4.5 & (e) & 157299.94 & 3.5 & (o) & -0.86 & $5.54 \mathrm{E}+08$ \\
\hline 128.8688 & 83897.98 & 2.5 & (e) & 161496.05 & 3.5 & (o) & -0.83 & $5.97 \mathrm{E}+08$ \\
\hline 128.9230 & 81303.91 & 6.5 & (e) & 158869.30 & 6.5 & (o) & -0.01 & $3.95 \mathrm{E}+09$ \\
\hline 129.0619 & 84092.98 & 6.5 & (e) & 161574.84 & 5.5 & (o) & 0.14 & $5.49 \mathrm{E}+09$ \\
\hline 129.1412 & 81832.25 & 3.5 & (e) & 159266.55 & 2.5 & (o) & -0.78 & $6.67 \mathrm{E}+08$ \\
\hline 129.2804 & 75666.91 & 3.5 & (e) & 153018.22 & 3.5 & (o) & -0.29 & $2.05 \mathrm{E}+09$ \\
\hline $129.2831 *$ & 81303.91 & 6.5 & (e) & 158653.53 & 7.5 & (o) & -0.99 & $4.12 \mathrm{E}+08$ \\
\hline 129.3031D & 78864.24 & 7.5 & (e) & 156201.33 & 6.5 & (o) & -0.18 & $2.61 \mathrm{E}+09$ \\
\hline 129.3031D & 102627.40 & 4.5 & (e) & 179964.22 & 3.5 & (o) & -0.14 & $2.89 \mathrm{E}+09$ \\
\hline 129.3286 & 83903.04 & 5.5 & (e) & 161225.30 & 4.5 & (o) & -0.16 & $2.79 \mathrm{E}+09$ \\
\hline 129.3559 & 78702.59 & 5.5 & (e) & 156008.48 & 4.5 & (o) & -0.27 & $2.15 E+09$ \\
\hline $129.4602 *$ & 84408.39 & 4.5 & (e) & 161652.20 & 3.5 & (o) & -0.79 & $6.51 \mathrm{E}+08$ \\
\hline 129.4853 & 102242.87 & 5.5 & (e) & 79471.41 & 5.5 & (o) & -0.04 & $3.61 \mathrm{E}+09$ \\
\hline 129.4951D & 80080.41 & 5.5 & (e) & 157302.95 & 5.5 & (o) & -0.91 & $4.82 \mathrm{E}+08$ \\
\hline $129.5813^{*}$ & 80128.30 & 2.5 & (e) & 157299.94 & 3.5 & (o) & -0.96 & $4.39 \mathrm{E}+08$ \\
\hline $129.5871 \mathrm{D}$ & 78702.59 & 5.5 & (e) & 155870.45 & 5.5 & (o) & -0.15 & $2.82 \mathrm{E}+09$ \\
\hline $129.5871 \mathrm{D}$ & 82669.72 & 4.5 & (e) & 159837.56 & 4.5 & (o) & -0.98 & $4.18 \mathrm{E}+08$ \\
\hline 129.6011 & 88642.61 & 4.5 & (e) & 165802.12 & 5.5 & (o) & -0.92 & $4.75 \mathrm{E}+08$ \\
\hline $129.6573 \mathrm{D}$ & 84775.85 & 1.5 & (e) & 161901.81 & 2.5 & (o) & -0.49 & $1.27 \mathrm{E}+09$ \\
\hline 129.6813 & 88642.61 & 4.5 & (e) & 165754.41 & 4.5 & (o) & -0.45 & $1.42 \mathrm{E}+09$ \\
\hline 129.7223 & 84408.39 & 4.5 & (e) & 161496.05 & 3.5 & (o) & -0.37 & $1.70 \mathrm{E}+09$ \\
\hline 129.7842D & 73556.74 & 5.5 & (e) & 150607.31 & 5.5 & (o) & 0.06 & $4.52 \mathrm{E}+09$ \\
\hline 129.7842D & 77809.61 & 4.5 & (e) & 154860.81 & 4.5 & (o) & -0.79 & $6.41 \mathrm{E}+08$ \\
\hline 129.7881 & 88135.24 & 2.5 & (e) & 165183.48 & 2.5 & (o) & -0.70 & $7.86 \mathrm{E}+08$ \\
\hline 129.8490 & 79189.02 & 6.5 & (e) & 156201.33 & 6.5 & (o) & -0.49 & $1.27 \mathrm{E}+09$ \\
\hline 129.8601 & 101159.67 & 6.5 & (e) & 178165.59 & 6.5 & (o) & 0.01 & $4.07 E+09$ \\
\hline 129.8634 & 73556.74 & 5.5 & (e) & 150560.55 & 4.5 & (o) & -0.08 & $3.31 \mathrm{E}+09$ \\
\hline $129.8990^{*}$ & 77598.12 & 2.5 & (e) & 154581.00 & 2.5 & (o) & -0.71 & 7.64E+08 \\
\hline 129.9302 & 83897.98 & 2.5 & (e) & 160862.38 & 2.5 & (o) & -0.59 & $1.02 \mathrm{E}+09$ \\
\hline 129.9798 & 76471.40 & 6.5 & (e) & 153406.25 & 5.5 & (o) & -0.30 & $1.98 \mathrm{E}+09$ \\
\hline 130.0121 & 82669.72 & 4.5 & (e) & 159585.52 & 3.5 & (o) & -0.42 & $1.50 \mathrm{E}+09$ \\
\hline 130.1068 & 88097.78 & 5.5 & (e) & 164957.81 & 4.5 & (o) & -0.38 & $1.65 \mathrm{E}+09$ \\
\hline 130.1688 & 84672.74 & 3.5 & (e) & 161496.05 & 3.5 & (o) & -0.66 & $8.64 \mathrm{E}+08$ \\
\hline 130.2499 & 81950.73 & 1.5 & (e) & 158725.98 & 1.5 & (o) & -0.97 & $4.21 \mathrm{E}+08$ \\
\hline 130.2960 & 77833.03 & 3.5 & (e) & 154581.00 & 2.5 & (o) & -0.44 & $1.43 \mathrm{E}+09$ \\
\hline 130.3315D & 70817.12 & 4.5 & (e) & 147544.89 & 4.5 & (o) & -0.004 & $3.89 \mathrm{E}+09$ \\
\hline 130.3315D & 81303.91 & 6.5 & (e) & 158032.30 & 5.5 & (o) & -0.24 & $2.29 \mathrm{E}+09$ \\
\hline $130.4085 \mathrm{D}$ & 79189.02 & 6.5 & (e) & 155870.45 & 5.5 & (o) & -0.76 & $6.84 \mathrm{E}+08$ \\
\hline 130.4085D & 85352.26 & 4.5 & (e) & 162034.11 & 4.5 & (o) & -0.79 & $6.42 \mathrm{E}+08$ \\
\hline 130.4994 & 97727.22 & 1.5 & (e) & 174355.70 & 1.5 & (o) & -0.79 & $6.40 \mathrm{E}+08$ \\
\hline 130.5372 & 76411.72 & 4.5 & (e) & 153018.22 & 3.5 & (o) & -0.94 & $4.57 \mathrm{E}+08$ \\
\hline 130.6183 & 74571.83 & 5.5 & (e) & 151130.44 & 4.5 & (o) & -0.80 & $6.19 \mathrm{E}+08$ \\
\hline 130.6294 & 84672.74 & 3.5 & (e) & 161225.30 & 4.5 & (o) & -0.73 & $7.27 \mathrm{E}+08$ \\
\hline 130.6889 & 83903.04 & 5.5 & (e) & 160420.64 & 4.5 & (o) & -0.68 & $8.24 \mathrm{E}+08$ \\
\hline 130.8946 & 83768.34 & 3.5 & (e) & 160165.53 & 3.5 & (o) & -0.99 & $3.97 \mathrm{E}+08$ \\
\hline 130.9058 & 84266.98 & 0.5 & (e) & 160657.91 & 1.5 & (o) & -0.60 & $9.77 \mathrm{E}+08$ \\
\hline 131.0311 & 85716.11 & 4.5 & (e) & 162034.11 & 4.5 & (o) & -0.85 & $5.55 \mathrm{E}+08$ \\
\hline 131.0363 & 88642.61 & 4.5 & (e) & 164957.81 & 4.5 & (o) & -0.76 & $6.72 \mathrm{E}+08$ \\
\hline 131.0557 & 22043.77 & 7.5 & (o) & 98347.09 & 6.5 & (e) & -0.84 & $5.61 \mathrm{E}+08$ \\
\hline 131.0616 & 85352.26 & 4.5 & (e) & 161652.20 & 3.5 & (o) & -0.98 & $4.08 \mathrm{E}+08$ \\
\hline 131.1951 & 85352.26 & 4.5 & (e) & 161574.84 & 5.5 & (o) & -0.85 & $5.47 \mathrm{E}+08$ \\
\hline 131.2969 & 84698.79 & 1.5 & (e) & 160862.38 & 2.5 & (o) & -0.72 & $7.30 \mathrm{E}+08$ \\
\hline 131.3690D & 76471.40 & 6.5 & (e) & 152592.62 & 5.5 & (o) & -0.75 & $6.88 \mathrm{E}+08$ \\
\hline 131.4293D & 84775.85 & 1.5 & (e) & 160862.38 & 2.5 & (o) & -0.63 & $9.11 \mathrm{E}+08$ \\
\hline 131.4588 & 83768.34 & 3.5 & (e) & 159837.56 & 4.5 & (o) & -0.81 & $5.98 \mathrm{E}+08$ \\
\hline $131.5015^{*}$ & 98541.35 & 0.5 & (e) & 174586.12 & 1.5 & (o) & -0.90 & $4.87 \mathrm{E}+08$ \\
\hline 131.5576 & 84408.39 & 4.5 & (e) & 160420.64 & 4.5 & (o) & -0.38 & $1.62 \mathrm{E}+09$ \\
\hline
\end{tabular}


Table 3. (Continued.)

\begin{tabular}{|c|c|c|c|c|c|c|c|c|}
\hline \multirow[b]{2}{*}{ Wavelength $^{\mathrm{a}}(\mathrm{nm})$} & \multicolumn{3}{|c|}{ Lower level $^{\mathrm{b}}$} & \multicolumn{3}{|c|}{ Upper level $^{\mathrm{b}}$} & \multirow[b]{2}{*}{$\log g f^{\mathrm{c}}$} & \multirow[b]{2}{*}{$g A^{\mathrm{c}}\left(\mathrm{s}^{-1}\right)$} \\
\hline & $\mathrm{E}\left(\mathrm{cm}^{-1}\right)$ & $J$ & Parity & $\mathrm{E}\left(\mathrm{cm}^{-1}\right)$ & $J$ & Parity & & \\
\hline 131.5666 & 84855.30 & 2.5 & (e) & 160862.38 & 2.5 & (o) & -0.76 & $6.67 \mathrm{E}+08$ \\
\hline 131.5807 & 81303.91 & 6.5 & (e) & 157302.95 & 5.5 & (o) & -0.89 & $5.02 \mathrm{E}+08$ \\
\hline 131.6498 & 84698.79 & 1.5 & (e) & 160657.91 & 1.5 & (o) & -0.58 & $1.02 \mathrm{E}+09$ \\
\hline 131.6941 & 74673.95 & 6.5 & (e) & 150607.31 & 5.5 & (o) & 0.22 & $6.44 \mathrm{E}+09$ \\
\hline 131.7119 & 102242.87 & 5.5 & (e) & 178165.59 & 6.5 & (o) & -0.48 & $1.28 \mathrm{E}+09$ \\
\hline 131.7990 & 85352.26 & 4.5 & (e) & 161225.30 & 4.5 & (o) & -0.52 & $1.16 \mathrm{E}+09$ \\
\hline 131.8669 & 86067.88 & 3.5 & (e) & 161901.81 & 2.5 & (o) & -0.95 & $4.27 \mathrm{E}+08$ \\
\hline 131.9249 & 71744.57 & 5.5 & (e) & 147544.89 & 4.5 & (o) & 0.28 & $7.29 \mathrm{E}+09$ \\
\hline 132.0004 & 84408.39 & 4.5 & (e) & 160165.53 & 3.5 & (o) & -0.54 & $1.11 \mathrm{E}+09$ \\
\hline 132.1108 & 83032.16 & 1.5 & (e) & 158725.98 & 1.5 & (o) & -0.73 & $7.19 \mathrm{E}+08$ \\
\hline $132.2610 \mathrm{D}$ & 99195.97 & 3.5 & (e) & 174803.78 & 2.5 & (o) & -0.31 & $1.87 \mathrm{E}+09$ \\
\hline 132.2715 & 98347.09 & 6.5 & (e) & 173949.03 & 6.5 & (o) & -0.10 & $3.00 \mathrm{E}+09$ \\
\hline 132.2809 & 77809.61 & 4.5 & (e) & 153406.25 & 5.5 & (o) & -0.70 & $7.67 \mathrm{E}+08$ \\
\hline 132.3021 & 86067.88 & 3.5 & (e) & 161652.20 & 3.5 & (o) & -0.78 & $6.29 \mathrm{E}+08$ \\
\hline 132.3570 & 99033.19 & 2.5 & (e) & 174586.12 & 1.5 & (o) & -0.85 & $5.41 \mathrm{E}+08$ \\
\hline 132.4334D & 85716.11 & 4.5 & (e) & 161225.30 & 4.5 & (o) & -0.79 & $6.16 \mathrm{E}+08$ \\
\hline 132.4334D & 104775.22 & 8.5 & (e) & 180284.95 & 7.5 & (o) & 0.49 & $1.18 \mathrm{E}+10$ \\
\hline 132.4611 & 77912.50 & 5.5 & (e) & 153406.25 & 5.5 & (o) & -0.07 & $3.22 \mathrm{E}+09$ \\
\hline $132.5755 \mathrm{D}$ & 84408.39 & 4.5 & (e) & 159837.56 & 4.5 & (o) & -0.95 & $4.31 \mathrm{E}+08$ \\
\hline 132.5879 & 90380.51 & 6.5 & (e) & 165802.12 & 5.5 & (o) & 0.22 & $6.26 \mathrm{E}+09$ \\
\hline 132.5906 & 77598.12 & 2.5 & (e) & 153018.22 & 3.5 & (o) & -0.31 & $1.83 \mathrm{E}+09$ \\
\hline 132.6090 & 102756.25 & 7.5 & (e) & 178165.59 & 6.5 & (o) & 0.28 & $7.24 \mathrm{E}+09$ \\
\hline 132.6363 & 99409.66 & 2.5 & (e) & 174803.78 & 2.5 & (o) & -0.93 & $4.42 \mathrm{E}+08$ \\
\hline 132.6918 & 82669.72 & 4.5 & (e) & 158032.30 & 5.5 & (o) & -0.84 & $5.50 \mathrm{E}+08$ \\
\hline 132.7626 & 99033.19 & 2.5 & (e) & 174355.70 & 1.5 & (o) & -0.70 & $7.50 \mathrm{E}+08$ \\
\hline 132.7749 & 5988.51 & 7.5 & (o) & 81303.91 & 6.5 & (e) & -0.41 & $1.45 \mathrm{E}+09$ \\
\hline 132.7841 & 84855.30 & 2.5 & (e) & 160165.53 & 3.5 & (o) & -0.80 & $6.02 \mathrm{E}+08$ \\
\hline 132.8500 & 98676.42 & 5.5 & (e) & 173949.03 & 6.5 & (o) & -0.68 & $7.83 \mathrm{E}+08$ \\
\hline 132.9048 & 79339.01 & 1.5 & (e) & 154581.00 & 2.5 & (o) & -0.62 & $9.12 \mathrm{E}+08$ \\
\hline $133.0204 \mathrm{D}$ & 99409.66 & 2.5 & (e) & 174586.12 & 1.5 & (o) & -0.83 & $5.57 \mathrm{E}+08$ \\
\hline 133.0901 & 83588.99 & 2.5 & (e) & 158725.98 & 1.5 & (o) & -0.76 & $6.53 \mathrm{E}+08$ \\
\hline 133.1248D & 79694.96 & 4.5 & (e) & 154812.25 & 3.5 & (o) & -0.12 & $2.87 \mathrm{E}+09$ \\
\hline 133.1248D & 81155.44 & 4.5 & (e) & 156272.69 & 3.5 & (o) & -0.38 & $1.57 \mathrm{E}+09$ \\
\hline 132.1398 & 83588.99 & 2.5 & (e) & 159266.55 & 2.5 & (o) & -0.61 & $9.33 \mathrm{E}+08$ \\
\hline $133.1652 *$ & 80663.34 & 1.5 & (e) & 155758.06 & 2.5 & (o) & -0.69 & $7.71 \mathrm{E}+08$ \\
\hline 133.1970D & 104888.37 & 3.5 & (e) & 179964.22 & 3.5 & (o) & -0.63 & $8.77 \mathrm{E}+08$ \\
\hline 133.3523 & 100976.62 & 3.5 & (e) & 175965.55 & 2.5 & (o) & -0.65 & $8.39 \mathrm{E}+08$ \\
\hline 133.3657 & 81155.44 & 4.5 & (e) & 156137.08 & 4.5 & (o) & -0.30 & $1.88 \mathrm{E}+09$ \\
\hline 133.4023 & 87072.95 & 5.5 & (e) & 162034.11 & 4.5 & (o) & -0.61 & $9.29 \mathrm{E}+08$ \\
\hline 133.4297 & 99409.66 & 2.5 & (e) & 174355.70 & 1.5 & (o) & -1.00 & $3.75 E+08$ \\
\hline $133.4468 *$ & 82363.72 & 3.5 & (e) & 157299.94 & 3.5 & (o) & -0.54 & $1.08 \mathrm{E}+09$ \\
\hline 133.4884 & 84672.74 & 3.5 & (e) & 159585.52 & 3.5 & (o) & -0.42 & $1.42 \mathrm{E}+09$ \\
\hline $133.5161 \mathrm{~T}$ & 79189.02 & 6.5 & (e) & 154086.17 & 5.5 & (o) & -0.61 & $9.18 \mathrm{E}+08$ \\
\hline $133.5161 \mathrm{~T}$ & 81375.35 & 3.5 & (e) & 156272.69 & 3.5 & (o) & -0.90 & $4.71 \mathrm{E}+08$ \\
\hline 133.5508 & 77833.03 & 3.5 & (e) & 152710.30 & 2.5 & (o) & -0.14 & $2.71 E+09$ \\
\hline 133.5225 & 75666.91 & 3.5 & (e) & 150560.55 & 4.5 & (o) & -0.42 & $1.42 \mathrm{E}+09$ \\
\hline 133.5248 & 90291.23 & 1.5 & (e) & 165183.48 & 2.5 & (o) & -0.18 & $2.49 \mathrm{E}+09$ \\
\hline 133.5420 & 91028.86 & 4.5 & (e) & 165911.59 & 3.5 & (o) & -0.50 & $1.18 \mathrm{E}+09$ \\
\hline 133.6009 & 81422.92 & 2.5 & (e) & 156272.69 & 3.5 & (o) & -0.22 & $2.26 \mathrm{E}+09$ \\
\hline 133.6119 & 90339.84 & 2.5 & (e) & 165183.48 & 2.5 & (o) & -0.43 & $1.40 \mathrm{E}+09$ \\
\hline 133.6399 & 83897.98 & 2.5 & (e) & 158725.98 & 1.5 & (o) & -0.86 & $5.20 \mathrm{E}+08$ \\
\hline $133.6498 *$ & 91028.86 & 4.5 & (e) & 165851.25 & 3.5 & (o) & -0.45 & $1.32 \mathrm{E}+09$ \\
\hline 133.6980 & 3907.43 & 6.5 & (o) & 78702.59 & 5.5 & (e) & -0.49 & $1.20 \mathrm{E}+09$ \\
\hline 133.7285 & 90380.51 & 6.5 & (e) & 165158.58 & 5.5 & (o) & -0.37 & $1.60 \mathrm{E}+09$ \\
\hline $133.7322 *$ & 84092.98 & 6.5 & (e) & 158869.30 & 6.5 & (o) & -0.93 & $4.39 \mathrm{E}+08$ \\
\hline 133.7583 & 81375.35 & 3.5 & (e) & 156137.08 & 4.5 & (o) & -0.63 & $8.64 \mathrm{E}+08$ \\
\hline $133.7883^{*}$ & 81013.13 & 1.5 & (e) & 155758.06 & 2.5 & (o) & -0.45 & $1.32 \mathrm{E}+09$ \\
\hline 133.7985 & 12800.29 & 4.5 & (o) & 87539.35 & 5.5 & (e) & -0.98 & $3.86 \mathrm{E}+08$ \\
\hline 133.8229 & 91028.86 & 4.5 & (e) & 165754.41 & 4.5 & (o) & -0.77 & $6.29 \mathrm{E}+08$ \\
\hline 133.8619D & 78702.59 & 5.5 & (e) & 153406.25 & 5.5 & (o) & -0.48 & $1.23 \mathrm{E}+09$ \\
\hline 133.8619D & 85716.11 & 4.5 & (e) & 160420.64 & 4.5 & (o) & -0.63 & $8.62 \mathrm{E}+08$ \\
\hline $133.9033 \mathrm{D}$ & 77912.50 & 5.5 & (e) & 152592.92 & 4.5 & (o) & -0.78 & $6.23 \mathrm{E}+08$ \\
\hline 133.9127 & 98347.09 & 6.5 & (e) & 173022.52 & 5.5 & (o) & -0.19 & $2.43 \mathrm{E}+09$ \\
\hline
\end{tabular}


Table 3. (Continued.)

\begin{tabular}{|c|c|c|c|c|c|c|c|c|}
\hline \multirow[b]{2}{*}{ Wavelength $^{\mathrm{a}}(\mathrm{nm})$} & \multicolumn{3}{|c|}{ Lower level $^{b}$} & \multicolumn{3}{|c|}{ Upper level $^{\mathrm{b}}$} & \multirow[b]{2}{*}{$\log g f^{\mathrm{c}}$} & \multirow[b]{2}{*}{$g A^{\mathrm{c}}\left(\mathrm{s}^{-1}\right)$} \\
\hline & $\mathrm{E}\left(\mathrm{cm}^{-1}\right)$ & $J$ & Parity & $\mathrm{E}\left(\mathrm{cm}^{-1}\right)$ & $J$ & Parity & & \\
\hline 133.9884 & 87400.57 & 3.5 & (e) & 162034.11 & 4.5 & (o) & -0.71 & $7.27 \mathrm{E}+08$ \\
\hline 134.0596 & 84672.74 & 3.5 & (e) & 159266.55 & 2.5 & (o) & -0.64 & $8.50 \mathrm{E}+08$ \\
\hline 134.1080 & 98347.09 & 6.5 & (e) & 172913.80 & 6.5 & (o) & -0.24 & $2.16 \mathrm{E}+09$ \\
\hline 134.2014 & 1897.11 & 5.5 & (o) & 76411.72 & 4.5 & (e) & -0.53 & $1.10 \mathrm{E}+09$ \\
\hline 134.3139 & 80128.30 & 2.5 & (e) & 154581.00 & 2.5 & (o) & -0.84 & $5.32 \mathrm{E}+08$ \\
\hline 134.3255 & 81691.09 & 5.5 & (e) & 156137.08 & 4.5 & (o) & -0.31 & $1.84 \mathrm{E}+09$ \\
\hline 134.3527 & 104888.37 & 3.5 & (e) & 179319.09 & 2.5 & (o) & -1.00 & $3.70 \mathrm{E}+08$ \\
\hline 134.4736 & 0.00 & 4.5 & (o) & 74363.99 & 3.5 & (e) & -0.59 & $9.37 \mathrm{E}+08$ \\
\hline $134.4765^{*}$ & 87539.35 & 3.5 & (e) & 161901.81 & 2.5 & (o) & -0.63 & $8.70 \mathrm{E}+08$ \\
\hline 134.5058 & 98676.42 & 5.5 & (e) & 173022.52 & 5.5 & (o) & 0.09 & $4.55 \mathrm{E}+09$ \\
\hline 134.5578 & 81691.09 & 5.5 & (e) & 156008.48 & 4.5 & (o) & -0.64 & $8.39 \mathrm{E}+08$ \\
\hline 134.5805D & 91497.12 & 5.5 & (e) & 165802.12 & 5.5 & (o) & -0.95 & $4.09 \mathrm{E}+08$ \\
\hline 134.6289 & 35136.61 & 5.5 & (o) & 109414.69 & 4.5 & (e) & -1.00 & $3.71 E+08$ \\
\hline 134.6782D & 80663.34 & 1.5 & (e) & 154914.81 & 2.5 & (o) & -1.00 & $3.69 \mathrm{E}+08$ \\
\hline 134.7030 & 98676.42 & 5.5 & (e) & 172913.80 & 6.5 & (o) & -0.82 & $5.56 \mathrm{E}+08$ \\
\hline 134.7104 & 85352.26 & 4.5 & (e) & 159585.52 & 3.5 & (o) & -0.57 & $9.80 \mathrm{E}+08$ \\
\hline 134.7396 & 79189.02 & 6.5 & (e) & 153406.25 & 5.5 & (o) & -0.41 & $1.44 \mathrm{E}+09$ \\
\hline 134.7792 & 76411.72 & 4.5 & (e) & 150607.31 & 5.5 & (o) & -0.45 & $1.31 \mathrm{E}+09$ \\
\hline 134.8574 & 87072.95 & 5.5 & (e) & 161225.30 & 4.5 & (o) & -0.53 & $1.08 \mathrm{E}+09$ \\
\hline 134.8637 & 76411.72 & 4.5 & (e) & 150560.55 & 4.5 & (o) & 0.03 & $3.96 \mathrm{E}+09$ \\
\hline 134.8872 & 76471.40 & 6.5 & (e) & 150607.31 & 5.5 & (o) & -0.78 & $6.04 \mathrm{E}+08$ \\
\hline 134.9134 & 85716.11 & 4.5 & (e) & 159837.56 & 4.5 & (o) & -0.37 & $1.55 \mathrm{E}+09$ \\
\hline 134.9568 & 86067.88 & 3.5 & (e) & 160165.53 & 3.5 & (o) & -0.44 & $1.34 \mathrm{E}+09$ \\
\hline 134.9589 & 85716.11 & 4.5 & (e) & 159812.28 & 5.5 & (o) & -0.74 & $6.67 \mathrm{E}+08$ \\
\hline 135.2142 & 87539.35 & 3.5 & (e) & 161496.05 & 3.5 & (o) & -0.68 & $7.61 \mathrm{E}+08$ \\
\hline 135.2505 & 88097.78 & 5.5 & (e) & 162034.11 & 4.5 & (o) & -0.85 & $5.23 \mathrm{E}+08$ \\
\hline 135.2706 & 81832.25 & 3.5 & (e) & 155758.06 & 2.5 & (o) & -0.97 & $3.90 \mathrm{E}+08$ \\
\hline 135.3014 & 82363.72 & 3.5 & (e) & 156272.69 & 3.5 & (o) & -0.45 & $1.29 \mathrm{E}+09$ \\
\hline 135.4559 & 87400.57 & 3.5 & (e) & 161225.30 & 4.5 & (o) & -0.99 & $3.67 \mathrm{E}+08$ \\
\hline $135.4879 *$ & 81950.73 & 1.5 & (e) & 155758.06 & 2.5 & (o) & -0.52 & $1.09 \mathrm{E}+09$ \\
\hline 135.5028 & 81013.13 & 2.5 & (e) & 154812.25 & 3.5 & (o) & -0.88 & $4.86 \mathrm{E}+08$ \\
\hline 135.5621 & 88135.24 & 2.5 & (e) & 161901.81 & 2.5 & (o) & -0.99 & $3.67 \mathrm{E}+08$ \\
\hline $135.6643 \mathrm{D}$ & 79307.06 & 3.5 & (e) & 153018.22 & 3.5 & (o) & -0.56 & $1.01 \mathrm{E}+09$ \\
\hline $135.7870 \mathrm{D}$ & 82363.72 & 3.5 & (e) & 156008.48 & 4.5 & (o) & -0.55 & $1.01 \mathrm{E}+09$ \\
\hline 135.9288 & 81013.13 & 2.5 & (e) & 154581.00 & 2.5 & (o) & -0.67 & $7.67 \mathrm{E}+08$ \\
\hline 135.9944D & 83768.34 & 3.5 & (e) & 157299.94 & 3.5 & (o) & -0.90 & $4.59 \mathrm{E}+08$ \\
\hline $136.0230 *$ & 88135.24 & 2.5 & (e) & 161652.20 & 3.5 & (o) & -0.59 & $9.31 E+08$ \\
\hline 136.0356 & 101075.79 & 0.5 & (e) & 174586.12 & 1.5 & (o) & -0.88 & $4.76 \mathrm{E}+08$ \\
\hline $136.1252 *$ & 87400.57 & 3.5 & (e) & 160862.38 & 2.5 & (o) & -0.99 & $3.68 \mathrm{E}+08$ \\
\hline $136.1713 *$ & 81375.35 & 3.5 & (e) & 154812.25 & 3.5 & (o) & -0.98 & $3.72 \mathrm{E}+08$ \\
\hline 136.2926 & 79339.01 & 1.5 & (e) & 152710.30 & 2.5 & (o) & -0.45 & $1.29 \mathrm{E}+09$ \\
\hline 136.3363 & 87072.95 & 5.5 & (e) & 160420.64 & 4.5 & (o) & -0.50 & $1.13 \mathrm{E}+09$ \\
\hline $136.3825 \mathrm{D}$ & 87539.35 & 3.5 & (e) & 160862.38 & 2.5 & (o) & -0.53 & $1.06 \mathrm{E}+09$ \\
\hline 136.4510 & 79307.06 & 3.5 & (e) & 152592.92 & 4.5 & (o) & -0.91 & $4.42 \mathrm{E}+08$ \\
\hline 136.6111 & 5988.51 & 7.5 & (o) & 79189.02 & 6.5 & (e) & -0.75 & $6.30 \mathrm{E}+08$ \\
\hline 136.6472 & 74363.99 & 3.5 & (e) & 147544.89 & 4.5 & (o) & -0.58 & $9.48 \mathrm{E}+08$ \\
\hline 136.7730 & 100835.39 & 7.5 & (e) & 173949.03 & 6.5 & (o) & -0.11 & $2.76 \mathrm{E}+09$ \\
\hline 136.8897 & 87114.16 & 2.5 & (e) & 160165.53 & 3.5 & (o) & -0.66 & $7.73 E+08$ \\
\hline 136.9486 & 87400.57 & 3.5 & (e) & 160420.64 & 4.5 & (o) & -0.63 & $8.28 \mathrm{E}+08$ \\
\hline 136.9503 & 92892.71 & 3.5 & (e) & 165911.59 & 3.5 & (o) & -0.49 & $1.15 \mathrm{E}+09$ \\
\hline 136.9679 & 88642.61 & 4.5 & (e) & 161652.20 & 3.5 & (o) & -0.89 & $4.63 E+08$ \\
\hline 137.0234 & 81832.25 & 3.5 & (e) & 154812.25 & 3.5 & (o) & -0.90 & $4.54 \mathrm{E}+08$ \\
\hline 137.0638 & 92892.71 & 3.5 & (e) & 165851.25 & 3.5 & (o) & -0.38 & $1.48 \mathrm{E}+09$ \\
\hline 137.2202 & 5988.51 & 7.5 & (o) & 78864.24 & 7.5 & (e) & -0.47 & $1.19 \mathrm{E}+09$ \\
\hline 137.2463 & 92892.71 & 3.5 & (e) & 165754.41 & 4.5 & (o) & -0.83 & $5.31 \mathrm{E}+08$ \\
\hline 137.4202 & 101586.15 & 2.5 & (e) & 174355.70 & 1.5 & (o) & -0.91 & $4.37 \mathrm{E}+08$ \\
\hline 137.4287D & 87072.95 & 5.5 & (e) & 159837.56 & 4.5 & (o) & -0.46 & $1.22 \mathrm{E}+09$ \\
\hline 137.4997 & 88135.24 & 2.5 & (e) & 160862.38 & 2.5 & (o) & -0.99 & $3.66 \mathrm{E}+08$ \\
\hline 137.5427 & 89329.54 & 4.5 & (e) & 162034.11 & 4.5 & (o) & -0.68 & $7.37 \mathrm{E}+08$ \\
\hline $137.5797 \mathrm{~T}$ & 83588.99 & 2.5 & (e) & 156272.69 & 3.5 & (o) & -0.68 & $7.47 \mathrm{E}+08$ \\
\hline $137.5797 \mathrm{~T}$ & 101900.99 & 1.5 & (e) & 174586.12 & 1.5 & (o) & -0.63 & $8.25 \mathrm{E}+08$ \\
\hline $137.6914 *$ & 87539.35 & 2.5 & (e) & 160165.53 & 3.5 & (o) & -0.68 & $7.34 \mathrm{E}+08$ \\
\hline 137.7741D & 88642.61 & 4.5 & (e) & 161225.30 & 4.5 & (o) & -0.77 & $5.92 \mathrm{E}+08$ \\
\hline $137.7785 *$ & 103385.28 & 2.5 & (e) & 175965.55 & 2.5 & (o) & -0.87 & $4.69 E+08$ \\
\hline
\end{tabular}


Table 3. (Continued.)

\begin{tabular}{|c|c|c|c|c|c|c|c|c|}
\hline \multirow[b]{2}{*}{ Wavelength $^{\mathrm{a}}(\mathrm{nm})$} & \multicolumn{3}{|c|}{ Lower level $^{b}$} & \multicolumn{3}{|c|}{ Upper level $^{\mathrm{b}}$} & \multirow[b]{2}{*}{$\log g f^{\mathrm{c}}$} & \multirow[b]{2}{*}{$g A^{\mathrm{c}}\left(\mathrm{s}^{-1}\right)$} \\
\hline & $\mathrm{E}\left(\mathrm{cm}^{-1}\right)$ & $J$ & Parity & $\mathrm{E}\left(\mathrm{cm}^{-1}\right)$ & $J$ & Parity & & \\
\hline 137.8093 & 3907.43 & 6.5 & (o) & 76471.40 & 6.5 & (e) & -0.56 & $9.73 \mathrm{E}+08$ \\
\hline 137.8137D & 83309.16 & 6.5 & (e) & 155870.45 & 5.5 & (o) & 0.04 & $3.83 \mathrm{E}+09$ \\
\hline 137.9224D & 83768.34 & 3.5 & (e) & 156272.69 & 3.5 & (o) & -0.55 & $9.99 \mathrm{E}+08$ \\
\hline 137.9671 & 16161.53 & 5.5 & (o) & 88642.61 & 4.5 & (e) & -0.88 & $4.60 \mathrm{E}+08$ \\
\hline 138.0363 & 84855.30 & 2.5 & (e) & 157299.94 & 3.5 & (o) & -0.68 & $7.26 \mathrm{E}+08$ \\
\hline 138.0509 & 87400.57 & 3.5 & (e) & 159837.56 & 4.5 & (o) & -0.38 & $1.43 \mathrm{E}+09$ \\
\hline 138.1809 & 83768.34 & 5.5 & (e) & 156137.08 & 4.5 & (o) & -0.72 & $6.71 \mathrm{E}+08$ \\
\hline 138.2687D & 89329.54 & 4.5 & (e) & 161652.20 & 3.5 & (o) & -0.91 & $4.32 \mathrm{E}+08$ \\
\hline 138.2814 & 85716.11 & 4.5 & (e) & 158032.30 & 5.5 & (o) & -0.46 & $1.22 \mathrm{E}+09$ \\
\hline 138.3309 & 92892.71 & 3.5 & (e) & 165183.48 & 2.5 & (o) & -0.69 & $7.14 \mathrm{E}+08$ \\
\hline 138.3327 & 91497.12 & 5.5 & (e) & 163786.31 & 4.5 & (o) & -0.94 & $3.98 \mathrm{E}+08$ \\
\hline $138.4068 *$ & 81155.44 & 6.5 & (e) & 153406.25 & 5.5 & (o) & -0.95 & $3.89 \mathrm{E}+08$ \\
\hline 138.4384 & 83903.04 & 5.5 & (e) & 156137.08 & 4.5 & (o) & -0.70 & $6.97 \mathrm{E}+08$ \\
\hline 138.4819 & 32563.57 & 8.5 & (o) & 104775.22 & 8.5 & (e) & -0.79 & $5.60 \mathrm{E}+08$ \\
\hline 138.5211 & 86678.25 & 7.5 & (e) & 158869.30 & 6.5 & (o) & 0.29 & $6.84 \mathrm{E}+09$ \\
\hline 138.5682 & 89329.54 & 4.5 & (e) & 161496.05 & 3.5 & (o) & -0.26 & $1.92 \mathrm{E}+09$ \\
\hline 138.7377 & 100835.39 & 7.5 & (e) & 172913.80 & 6.5 & (o) & -0.25 & $1.98 \mathrm{E}+09$ \\
\hline 138.8308D & 88135.24 & 2.5 & (e) & 160165.53 & 3.5 & (o) & -0.48 & $1.15 \mathrm{E}+09$ \\
\hline 139.1540 & 101159.67 & 6.5 & (e) & 173022.52 & 5.5 & (o) & -0.72 & $6.57 \mathrm{E}+08$ \\
\hline 139.1621 & 30179.93 & 5.5 & (o) & 102038.54 & 4.5 & (e) & -0.76 & $5.96 \mathrm{E}+08$ \\
\hline 139.3193 & 84092.98 & 6.5 & (e) & 155870.45 & 5.5 & (o) & -0.43 & $1.29 \mathrm{E}+09$ \\
\hline 139.4304 & 31036.00 & 7.5 & (o) & 102756.25 & 7.5 & (e) & -0.95 & $3.81 \mathrm{E}+08$ \\
\hline 139.4575 & 102242.87 & 5.5 & (e) & 173949.03 & 6.5 & (o) & -0.84 & $4.90 \mathrm{E}+08$ \\
\hline $139.5486 *$ & 1897.11 & 5.5 & (o) & 73556.74 & 5.5 & (e) & -0.75 & $6.08 \mathrm{E}+08$ \\
\hline 139.7393 & 90339.84 & 2.5 & (e) & 161901.81 & 2.5 & (o) & -0.61 & $8.52 \mathrm{E}+08$ \\
\hline 139.7648 & 83032.16 & 1.5 & (e) & 154581.00 & 2.5 & (o) & -0.89 & $4.41 \mathrm{E}+08$ \\
\hline $140.0201 \mathrm{~T}$ & 107900.68 & 3.5 & (e) & 179319.09 & 2.5 & (o) & -0.44 & $1.23 \mathrm{E}+09$ \\
\hline 140.0571 & 21493.39 & 4.5 & (o) & 92892.71 & 3.5 & (e) & -0.85 & $4.80 \mathrm{E}+08$ \\
\hline 140.2287 & 90339.84 & 2.5 & (e) & 161652.20 & 3.5 & (o) & -0.96 & $3.70 \mathrm{E}+08$ \\
\hline 140.3436 & 79307.06 & 3.5 & (e) & 150560.55 & 4.5 & (o) & -0.46 & $1.16 \mathrm{E}+09$ \\
\hline 140.4599D & 88642.61 & 4.5 & (e) & 159837.56 & 4.5 & (o) & -0.61 & $8.24 \mathrm{E}+08$ \\
\hline 140.4599D & 90380.51 & 6.5 & (e) & 161574.84 & 5.5 & (o) & -0.77 & $5.81 \mathrm{E}+08$ \\
\hline $140.4637 *$ & 102756.25 & 7.5 & (e) & 173949.03 & 6.5 & (o) & -0.67 & $7.23 \mathrm{E}+08$ \\
\hline 140.8766 & 102038.54 & 4.5 & (e) & 173022.52 & 5.5 & (o) & -0.31 & $1.63 \mathrm{E}+09$ \\
\hline $140.9038 *$ & 103385.28 & 2.5 & (e) & 174355.70 & 1.5 & (o) & -0.90 & $4.20 \mathrm{E}+08$ \\
\hline 141.1708 & 89329.54 & 4.5 & (e) & 160165.53 & 3.5 & (o) & -0.99 & $3.40 \mathrm{E}+08$ \\
\hline 141.2089 & 0.00 & 4.5 & (o) & 70817.12 & 4.5 & (e) & -0.82 & $5.12 \mathrm{E}+08$ \\
\hline 141.2886 & 83309.16 & 6.5 & (e) & 154086.17 & 5.5 & (o) & -0.09 & $2.72 \mathrm{E}+09$ \\
\hline 141.3698 & 82669.72 & 4.5 & (e) & 153406.25 & 5.5 & (o) & -0.12 & $2.55 \mathrm{E}+09$ \\
\hline 141.5010 & 102242.87 & 5.5 & (e) & 172913.80 & 6.5 & (o) & -0.97 & $3.58 \mathrm{E}+08$ \\
\hline 141.5221 & 31582.85 & 6.5 & (o) & 102242.87 & 5.5 & (e) & -0.53 & $9.94 \mathrm{E}+08$ \\
\hline 141.5963 & 91028.86 & 4.5 & (e) & 161652.20 & 3.5 & (o) & -0.80 & $5.25 \mathrm{E}+08$ \\
\hline 141.7695 & 91497.12 & 5.5 & (e) & 162034.11 & 4.5 & (o) & 0.01 & $3.42 \mathrm{E}+09$ \\
\hline 141.9101 & 91028.86 & 4.5 & (e) & 161496.05 & 3.5 & (o) & -0.99 & $3.38 \mathrm{E}+08$ \\
\hline 142.0552 & 102627.40 & 4.5 & (e) & 173022.52 & 5.5 & (o) & -0.62 & $7.91 \mathrm{E}+08$ \\
\hline 142.3369 & 89329.54 & 4.5 & (e) & 159585.52 & 3.5 & (o) & -0.46 & $1.15 \mathrm{E}+09$ \\
\hline 142.4646 & 32563.57 & 8.5 & (o) & 102756.25 & 7.5 & (e) & -0.92 & $3.98 \mathrm{E}+08$ \\
\hline 142.5362 & 102756.25 & 7.5 & (e) & 172913.80 & 6.5 & (o) & -0.82 & $4.97 \mathrm{E}+08$ \\
\hline 142.6048 & 31036.00 & 7.5 & (o) & 101159.67 & 6.5 & (e) & -0.31 & $1.59 \mathrm{E}+09$ \\
\hline 142.7419 & 109414.69 & 4.5 & (e) & 79471.41 & 5.5 & (o) & -0.02 & $3.15 E+09$ \\
\hline 143.1242 & 13719.82 & 3.5 & (o) & 83588.99 & 2.5 & (e) & -0.85 & $4.58 \mathrm{E}+08$ \\
\hline 143.4145 & 91497.12 & 5.5 & (e) & 161225.30 & 4.5 & (o) & -0.49 & $1.04 \mathrm{E}+09$ \\
\hline 143.5176 & 14994.87 & 4.5 & (o) & 84672.74 & 3.5 & (e) & -0.84 & $4.63 \mathrm{E}+08$ \\
\hline 143.8372 & 86678.25 & 7.5 & (e) & 156201.33 & 6.5 & (o) & -0.60 & $8.02 \mathrm{E}+08$ \\
\hline 143.8789 & 83903.04 & 5.5 & (e) & 153406.25 & 5.5 & (o) & -0.95 & $3.61 E+08$ \\
\hline $144.1136 *$ & 88642.61 & 4.5 & (e) & 158032.30 & 5.5 & (o) & -1.00 & $3.26 \mathrm{E}+08$ \\
\hline 144.5283 & 16161.53 & 5.5 & (o) & 85352.26 & 4.5 & (e) & -0.82 & $4.81 E+08$ \\
\hline 144.6414 & 91028.86 & 4.5 & (e) & 160165.53 & 3.5 & (o) & -0.87 & $4.33 \mathrm{E}+08$ \\
\hline 144.7932 & 87072.95 & 5.5 & (e) & 156137.08 & 4.5 & (o) & -0.61 & $7.77 \mathrm{E}+08$ \\
\hline 145.3308 & 91028.86 & 4.5 & (e) & 159837.56 & 4.5 & (o) & -0.85 & $4.45 E+08$ \\
\hline 145.5008 & 81832.25 & 3.5 & (e) & 150560.55 & 4.5 & (o) & -0.57 & $8.55 \mathrm{E}+08$ \\
\hline 145.9929 & 30179.93 & 5.5 & (o) & 98676.42 & 5.5 & (e) & -0.91 & $3.88 \mathrm{E}+08$ \\
\hline 146.0099 & 90380.51 & 6.5 & (e) & 158869.30 & 6.5 & (o) & -0.79 & $5.04 \mathrm{E}+08$ \\
\hline 146.2951 & 12800.29 & 4.5 & (o) & 81155.44 & 4.5 & (e) & -0.88 & $4.08 \mathrm{E}+08$ \\
\hline
\end{tabular}


Table 3. (Continued.)

\begin{tabular}{|c|c|c|c|c|c|c|c|c|}
\hline \multirow[b]{2}{*}{ Wavelength $^{\mathrm{a}}(\mathrm{nm})$} & \multicolumn{3}{|c|}{ Lower level $^{b}$} & \multicolumn{3}{|c|}{ Upper level $^{\mathrm{b}}$} & \multirow[b]{2}{*}{$\log g f^{\mathrm{c}}$} & \multirow[b]{2}{*}{$g A^{\mathrm{c}}\left(\mathrm{s}^{-1}\right)$} \\
\hline & $\mathrm{E}\left(\mathrm{cm}^{-1}\right)$ & $J$ & Parity & $\mathrm{E}\left(\mathrm{cm}^{-1}\right)$ & $J$ & Parity & & \\
\hline $146.3262 *$ & 91497.12 & 4.5 & (e) & 159837.56 & 4.5 & (o) & -0.79 & $5.00 \mathrm{E}+08$ \\
\hline 146.3342 & 22043.77 & 7.5 & (o) & 90380.51 & 6.5 & (e) & -0.29 & $1.60 \mathrm{E}+09$ \\
\hline 146.4733 & 32563.57 & 8.5 & (o) & 100835.39 & 7.5 & (e) & -0.12 & $2.35 \mathrm{E}+09$ \\
\hline 146.8597 & 20005.22 & 6.5 & (o) & 88097.78 & 5.5 & (e) & -0.76 & $5.34 \mathrm{E}+08$ \\
\hline 146.9186 & 107900.68 & 3.5 & (e) & 175965.55 & 2.5 & (o) & -0.73 & $5.76 \mathrm{E}+08$ \\
\hline 146.9413D & 31355.04 & 3.5 & (o) & 99409.66 & 2.5 & (e) & -0.94 & $3.57 \mathrm{E}+08$ \\
\hline 146.9413D & 85352.26 & 4.5 & (e) & 153406.25 & 5.5 & (o) & -0.61 & $7.56 \mathrm{E}+08$ \\
\hline 146.9747 & 88097.78 & 5.5 & (e) & 156137.08 & 4.5 & (o) & -0.97 & $3.29 \mathrm{E}+08$ \\
\hline 147.7652 & 14994.87 & 4.5 & (o) & 82669.72 & 4.5 & (e) & -0.79 & $4.92 \mathrm{E}+08$ \\
\hline 148.1685 & 35136.61 & 5.5 & (o) & 102627.40 & 4.5 & (e) & -0.89 & $3.93 \mathrm{E}+08$ \\
\hline 148.5636 & 31036.00 & 7.5 & (o) & 98347.09 & 6.5 & (e) & -0.53 & $9.00 \mathrm{E}+08$ \\
\hline 148.5923 & 83309.16 & 6.5 & (e) & 150607.31 & 5.5 & (o) & -0.61 & $7.39 \mathrm{E}+08$ \\
\hline 149.1032 & 20005.22 & 6.5 & (o) & 87072.95 & 5.5 & (e) & -0.50 & $6.22 \mathrm{E}+08$ \\
\hline 150.1613 & 22047.39 & 5.5 & (o) & 88642.61 & 4.5 & (e) & -0.77 & $5.03 E+08$ \\
\hline 151.2907 & 19969.79 & 4.5 & (o) & 86067.88 & 3.5 & (e) & -0.99 & $2.99 \mathrm{E}+08$ \\
\hline 154.7165 & 22043.77 & 7.5 & (o) & 86678.25 & 7.5 & (e) & -0.75 & $4.94 \mathrm{E}+08$ \\
\hline 155.1864 & 19969.79 & 4.5 & (o) & 84408.39 & 4.5 & (e) & -0.98 & $2.93 \mathrm{E}+08$ \\
\hline 155.7078 & 21493.39 & 4.5 & (o) & 85716.11 & 4.5 & (e) & -0.81 & $4.30 \mathrm{E}+08$ \\
\hline $156.2088 *$ & 98017.24 & 4.5 & (e) & 162034.11 & 4.5 & (o) & -0.88 & $3.65 \mathrm{E}+08$ \\
\hline 156.7342 & 24333.10 & 2.5 & (o) & 88135.24 & 2.5 & (e) & -0.98 & $2.84 \mathrm{E}+08$ \\
\hline 157.2139 & 109414.69 & 4.5 & (e) & 173022.52 & 5.5 & (o) & -0.53 & $8.08 \mathrm{E}+08$ \\
\hline 157.9678 & 20005.22 & 6.5 & (o) & 83309.16 & 6.5 & (e) & -0.98 & $2.78 \mathrm{E}+08$ \\
\hline 159.0324 & 35136.61 & 5.5 & (o) & 98017.24 & 4.5 & (e) & -0.69 & $5.35 \mathrm{E}+08$ \\
\hline 159.1871 & 33741.15 & 3.5 & (o) & 96560.31 & 3.5 & (e) & -0.63 & $6.16 \mathrm{E}+08$ \\
\hline 163.2244 & 22043.77 & 7.5 & (o) & 83309.16 & 6.5 & (e) & -0.87 & $3.38 \mathrm{E}+08$ \\
\hline 166.9054 & 31582.85 & 6.5 & (o) & 91497.12 & 5.5 & (e) & -0.42 & $9.09 \mathrm{E}+08$ \\
\hline 169.0625 & 30179.93 & 5.5 & (o) & 89329.54 & 4.5 & (e) & -0.57 & $6.33 \mathrm{E}+08$ \\
\hline 170.2756 & 49172.45 & 4.5 & (o) & 107900.68 & 3.5 & (e) & -0.45 & $8.17 \mathrm{E}+08$ \\
\hline $172.3130 *$ & 100835.39 & 7.5 & (e) & 158869.30 & 6.5 & (o) & -0.85 & $3.19 \mathrm{E}+08$ \\
\hline 172.4261 & 50160.95 & 3.5 & (o) & 108156.67 & 2.5 & (e) & -0.53 & $6.60 \mathrm{E}+08$ \\
\hline 177.4297 & 35136.61 & 5.5 & (o) & 91497.12 & 5.5 & (e) & -0.85 & $2.96 \mathrm{E}+08$ \\
\hline 184.7928 & 32563.57 & 8.5 & (o) & 86678.25 & 7.5 & (e) & -0.50 & $6.22 \mathrm{E}+08$ \\
\hline 191.3024 & 31036.00 & 7.5 & (o) & 83309.16 & 6.5 & (e) & -0.79 & $2.94 \mathrm{E}+08$ \\
\hline $214.0192 *$ & 118448.53 & 4.5 & (e) & 165158.58 & 5.5 & (o) & -0.71 & $2.80 \mathrm{E}+08$ \\
\hline 218.2341 & 115767.20 & 6.5 & (e) & 161574.84 & 5.5 & (o) & -0.62 & $3.34 \mathrm{E}+08$ \\
\hline 218.5090 & 113118.93 & 5.5 & (e) & 158869.30 & 6.5 & (o) & -0.88 & $1.85 \mathrm{E}+08$ \\
\hline 220.5014 & 116697.38 & 5.5 & (e) & 162034.11 & 4.5 & (o) & -0.35 & $6.19 \mathrm{E}+08$ \\
\hline $220.9923 *$ & 110634.11 & 4.5 & (e) & 155870.45 & 5.5 & (o) & -0.52 & $4.11 \mathrm{E}+08$ \\
\hline $222.5816^{*}$ & 113118.93 & 5.5 & (e) & 158032.30 & 5.5 & (o) & -0.87 & $1.84 \mathrm{E}+08$ \\
\hline $222.7597 *$ & 116697.38 & 5.5 & (e) & 161574.84 & 5.5 & (o) & -0.74 & $2.44 \mathrm{E}+08$ \\
\hline 223.5000 & 110634.11 & 4.5 & (e) & 155363.06 & 5.5 & (o) & -0.47 & $4.57 \mathrm{E}+08$ \\
\hline 224.5073 & 116697.38 & 5.5 & (e) & 161225.30 & 4.5 & (o) & -0.56 & $3.66 \mathrm{E}+08$ \\
\hline 224.5252 & 110056.47 & 3.5 & (e) & 154581.00 & 2.5 & (o) & 0.18 & $2.00 \mathrm{E}+09$ \\
\hline 224.8424 & 110056.47 & 3.5 & (e) & 154518.38 & 3.5 & (o) & -0.76 & $2.30 \mathrm{E}+08$ \\
\hline 225.1895 & 122515.64 & 4.5 & (e) & 166908.81 & 4.5 & (o) & -0.77 & $2.27 \mathrm{E}+08$ \\
\hline $225.7510 *$ & 116375.04 & 2.5 & (e) & 160657.91 & 1.5 & (o) & -0.94 & $1.50 \mathrm{E}+08$ \\
\hline 226.1341 & 68803.16 & 3.5 & (o) & 113010.89 & 2.5 & (e) & -0.83 & $1.93 \mathrm{E}+08$ \\
\hline 226.9218 & 122854.37 & 3.5 & (e) & 166908.81 & 4.5 & (o) & -0.74 & $2.36 \mathrm{E}+08$ \\
\hline 226.9695 & 115767.20 & 6.5 & (e) & 159812.28 & 5.5 & (o) & -0.26 & $7.04 \mathrm{E}+08$ \\
\hline 227.3630 & 136316.05 & 6.5 & (e) & 180284.95 & 7.5 & (o) & 0.79 & $8.00 \mathrm{E}+09$ \\
\hline 228.0413 & 113461.85 & 4.5 & (e) & 157299.94 & 3.5 & (o) & 0.19 & $2.01 \mathrm{E}+09$ \\
\hline 228.2891 & 116375.04 & 2.5 & (e) & 160165.53 & 3.5 & (o) & -0.48 & $4.21 \mathrm{E}+08$ \\
\hline 128.3275 & 102038.54 & 4.5 & (e) & 179964.22 & 3.5 & (o) & -0.44 & $1.46 \mathrm{E}+09$ \\
\hline 228.6387 & 116697.38 & 5.5 & (e) & 160420.64 & 4.5 & (o) & -0.37 & $5.42 \mathrm{E}+08$ \\
\hline 229.3627 & 118448.53 & 4.5 & (e) & 162034.11 & 4.5 & (o) & 0.11 & $1.66 \mathrm{E}+09$ \\
\hline 229.3756 & 117912.91 & 3.5 & (e) & 161496.05 & 3.5 & (o) & 0.27 & $2.33 \mathrm{E}+09$ \\
\hline 229.4351 & 110056.47 & 3.5 & (e) & 153628.30 & 4.5 & (o) & 0.04 & $1.37 \mathrm{E}+09$ \\
\hline 230.0682 & 110634.11 & 4.5 & (e) & 154086.17 & 5.5 & (o) & 0.60 & $4.99 \mathrm{E}+09$ \\
\hline 230.2417 & 118482.80 & 2.5 & (e) & 161901.81 & 2.5 & (o) & -0.02 & $1.20 \mathrm{E}+09$ \\
\hline $230.3665 \mathrm{D}$ & 122515.64 & 4.5 & (e) & 165911.59 & 3.5 & (o) & -0.46 & $4.37 \mathrm{E}+08$ \\
\hline 230.5463 & 115904.51 & 1.5 & (e) & 159266.55 & 2.5 & (o) & -0.12 & $9.56 \mathrm{E}+08$ \\
\hline 230.6859 & 122515.64 & 4.5 & (e) & 165851.25 & 3.5 & (o) & -0.37 & $5.41 \mathrm{E}+08$ \\
\hline 230.8096 & 117912.91 & 3.5 & (e) & 161225.30 & 4.5 & (o) & 0.33 & $2.66 \mathrm{E}+09$ \\
\hline 230.9481 & 122515.64 & 4.5 & (e) & 165802.12 & 5.5 & (o) & 0.64 & $5.39 \mathrm{E}+09$ \\
\hline
\end{tabular}


Table 3. (Continued.)

\begin{tabular}{|c|c|c|c|c|c|c|c|c|}
\hline \multirow[b]{2}{*}{ Wavelength $^{\mathrm{a}}(\mathrm{nm})$} & \multicolumn{3}{|c|}{ Lower level $^{b}$} & \multicolumn{3}{|c|}{ Upper level $^{\mathrm{b}}$} & \multirow[b]{2}{*}{$\log g f^{\mathrm{c}}$} & \multirow[b]{2}{*}{$g A^{\mathrm{c}}\left(\mathrm{s}^{-1}\right)$} \\
\hline & $\mathrm{E}\left(\mathrm{cm}^{-1}\right)$ & $J$ & Parity & $\mathrm{E}\left(\mathrm{cm}^{-1}\right)$ & $J$ & Parity & & \\
\hline 231.2031 & 122515.64 & 4.5 & (e) & 165754.41 & 4.5 & (o) & 0.16 & $1.79 \mathrm{E}+09$ \\
\hline 231.3537 & 116375.04 & 2.5 & (e) & 159585.52 & 3.5 & (o) & 0.38 & $3.00 \mathrm{E}+09$ \\
\hline 231.3899 & 118448.53 & 4.5 & (e) & 161652.20 & 3.5 & (o) & -0.33 & $5.79 \mathrm{E}+08$ \\
\hline 231.5761 & 118482.80 & 2.5 & (e) & 161652.20 & 3.5 & (o) & -0.74 & $2.27 \mathrm{E}+08$ \\
\hline $231.6496 \mathrm{D}$ & 136316.05 & 6.5 & (e) & 79471.41 & 5.5 & (o) & 0.30 & $2.49 \mathrm{E}+09$ \\
\hline 231.6496D & 136316.06 & 5.5 & (e) & 79471.41 & 5.5 & (o) & 0.07 & $1.44 \mathrm{E}+09$ \\
\hline 231.7312 & 116697.38 & 5.5 & (e) & 159837.56 & 4.5 & (o) & 0.41 & $3.16 \mathrm{E}+09$ \\
\hline 231.8065 & 118448.53 & 4.5 & (e) & 161574.84 & 5.5 & (o) & 0.59 & $4.78 \mathrm{E}+09$ \\
\hline 231.8675 & 116697.38 & 5.5 & (e) & 159812.28 & 5.5 & (o) & -0.01 & $1.20 \mathrm{E}+09$ \\
\hline 231.9360 & 115767.20 & 6.5 & (e) & 158869.30 & 6.5 & (o) & 0.32 & $2.55 \mathrm{E}+09$ \\
\hline 232.0426 & 113118.93 & 5.5 & (e) & 156201.33 & 6.5 & (o) & 0.67 & $5.72 \mathrm{E}+09$ \\
\hline 232.1782 & 122854.37 & 3.5 & (e) & 165911.59 & 3.5 & (o) & 0.05 & $1.40 \mathrm{E}+09$ \\
\hline $232.2301 *$ & 118448.53 & 4.5 & (e) & 161496.05 & 3.5 & (o) & -1.00 & $1.23 \mathrm{E}+08$ \\
\hline 232.3877 & 113118.93 & 5.5 & (e) & 156137.08 & 4.5 & (o) & -0.42 & $4.66 \mathrm{E}+08$ \\
\hline 232.4154 & 118482.80 & 2.5 & (e) & 161496.05 & 3.5 & (o) & 0.13 & $1.67 \mathrm{E}+09$ \\
\hline 232.5037 & 122854.37 & 3.5 & (e) & 165851.25 & 3.5 & (o) & 0.18 & $1.86 \mathrm{E}+09$ \\
\hline 232.5185 & 110634.11 & 4.5 & (e) & 153628.30 & 4.5 & (o) & -0.33 & $5.67 \mathrm{E}+08$ \\
\hline 232.6943 & 110056.47 & 3.5 & (e) & 153018.22 & 3.5 & (o) & 0.26 & $2.24 \mathrm{E}+09$ \\
\hline 232.7600 & 117912.91 & 3.5 & (e) & 160862.38 & 2.5 & (o) & -0.19 & $7.92 \mathrm{E}+08$ \\
\hline $233.0273 \mathrm{D}$ & 119133.54 & 3.5 & (e) & 162034.11 & 4.5 & (o) & 0.28 & $2.36 \mathrm{E}+09$ \\
\hline $233.0273 \mathrm{D}$ & 122854.37 & 3.5 & (e) & 165754.41 & 4.5 & (o) & 0.05 & $1.38 \mathrm{E}+09$ \\
\hline 233.0760 & 116375.04 & 2.5 & (e) & 159266.55 & 2.5 & (o) & 0.06 & $1.42 \mathrm{E}+09$ \\
\hline 233.0859 & 113118.93 & 5.5 & (e) & 156008.48 & 4.5 & (o) & -0.10 & $9.66 \mathrm{E}+08$ \\
\hline 233.1033 & 115767.20 & 6.5 & (e) & 158653.53 & 7.5 & (o) & 0.70 & $6.23 \mathrm{E}+09$ \\
\hline 233.4554 & 115904.51 & 1.5 & (e) & 158725.98 & 1.5 & (o) & 0.07 & $1.45 \mathrm{E}+09$ \\
\hline 233.7007 & 118448.53 & 4.5 & (e) & 161225.30 & 4.5 & (o) & -0.15 & $8.60 \mathrm{E}+08$ \\
\hline $233.7254 *$ & 110634.11 & 4.5 & (e) & 153406.25 & 5.5 & (o) & -0.87 & $1.63 \mathrm{E}+08$ \\
\hline 233.7462 & 119133.54 & 2.5 & (e) & 161901.81 & 2.5 & (o) & 0.07 & $1.43 \mathrm{E}+09$ \\
\hline 233.8384 & 113118.93 & 5.5 & (e) & 155870.45 & 5.5 & (o) & 0.25 & $2.19 \mathrm{E}+09$ \\
\hline 234.2564 & 113461.85 & 4.5 & (e) & 156137.08 & 4.5 & (o) & -0.12 & $9.14 \mathrm{E}+08$ \\
\hline 234.3738 & 110056.47 & 3.5 & (e) & 152710.30 & 2.5 & (o) & -0.02 & $1.17 \mathrm{E}+09$ \\
\hline 234.4339 & 122515.64 & 4.5 & (e) & 165158.58 & 5.5 & (o) & -0.02 & $1.17 \mathrm{E}+09$ \\
\hline 234.9646 & 113461.85 & 4.5 & (e) & 156008.48 & 4.5 & (o) & 0.09 & $1.47 \mathrm{E}+09$ \\
\hline 235.0191 & 110056.47 & 3.5 & (e) & 152592.92 & 4.5 & (o) & -0.09 & $9.91 \mathrm{E}+08$ \\
\hline 235.1193 & 119133.54 & 3.5 & (e) & 161652.20 & 3.5 & (o) & 0.26 & $2.22 \mathrm{E}+09$ \\
\hline 235.1791D & 117912.91 & 3.5 & (e) & 160420.64 & 4.5 & (o) & 0.11 & $1.54 \mathrm{E}+09$ \\
\hline 235.1791D & 137456.41 & 3.5 & (e) & 179964.22 & 3.5 & (o) & 0.32 & $2.54 \mathrm{E}+09$ \\
\hline 235.5433 & 122515.64 & 4.5 & (e) & 164957.81 & 4.5 & (o) & -0.14 & $8.68 \mathrm{E}+08$ \\
\hline 235.7298 & 113461.85 & 4.5 & (e) & 155870.45 & 5.5 & (o) & 0.40 & $3.01 \mathrm{E}+09$ \\
\hline 235.8658 & 110634.11 & 4.5 & (e) & 153018.22 & 3.5 & (o) & 0.10 & $1.50 \mathrm{E}+09$ \\
\hline 235.8904 & 118482.80 & 2.5 & (e) & 160862.38 & 2.5 & (o) & 0.05 & $1.34 \mathrm{E}+09$ \\
\hline 235.9849 & 119133.54 & 3.5 & (e) & 161496.05 & 3.5 & (o) & -0.98 & $1.26 \mathrm{E}+08$ \\
\hline 236.0504 & 116375.04 & 2.5 & (e) & 158725.98 & 1.5 & (o) & -0.63 & $2.83 \mathrm{E}+08$ \\
\hline 236.1722 & 122854.37 & 3.5 & (e) & 165183.48 & 2.5 & (o) & 0.38 & $2.87 \mathrm{E}+09$ \\
\hline 236.5294 & 115767.20 & 6.5 & (e) & 158032.30 & 5.5 & (o) & -0.002 & $1.21 \mathrm{E}+09$ \\
\hline 236.6471 & 113118.93 & 5.5 & (e) & 155363.06 & 5.5 & (o) & -0.78 & $2.00 \mathrm{E}+08$ \\
\hline 237.0342 & 118482.80 & 2.5 & (e) & 160657.91 & 1.5 & (o) & 0.04 & $1.31 \mathrm{E}+09$ \\
\hline 237.0513 & 116697.38 & 5.5 & (e) & 158869.30 & 6.5 & (o) & 0.54 & $4.11 \mathrm{E}+09$ \\
\hline 237.4371 & 122854.37 & 3.5 & (e) & 164957.81 & 4.5 & (o) & -0.30 & $5.89 \mathrm{E}+08$ \\
\hline 238.2556D & 110634.11 & 4.5 & (e) & 152592.62 & 5.5 & (o) & -0.36 & $5.17 \mathrm{E}+08$ \\
\hline 238.2556D & 110634.11 & 4.5 & (e) & 152592.92 & 4.5 & (o) & 0.11 & $1.50 \mathrm{E}+09$ \\
\hline 238.5840 & 113461.85 & 4.5 & (e) & 155363.06 & 5.5 & (o) & -0.49 & $3.82 \mathrm{E}+08$ \\
\hline 238.8787D & 136316.05 & 6.5 & (e) & 178165.59 & 6.5 & (o) & 0.18 & $1.77 \mathrm{E}+09$ \\
\hline 238.8787D & 136316.06 & 5.5 & (e) & 178165.59 & 6.5 & (o) & 0.49 & $3.66 \mathrm{E}+09$ \\
\hline 239.4951 & 113118.93 & 5.5 & (e) & 154860.81 & 4.5 & (o) & -0.67 & $2.48 \mathrm{E}+08$ \\
\hline 239.5698 & 119133.54 & 3.5 & (e) & 160862.38 & 2.5 & (o) & -0.15 & $8.16 \mathrm{E}+08$ \\
\hline 239.8927* & 117912.91 & 3.5 & (e) & 159585.52 & 3.5 & (o) & -0.71 & $2.25 \mathrm{E}+08$ \\
\hline 240.6841 & 115767.20 & 6.5 & (e) & 157302.95 & 5.5 & (o) & -0.83 & $1.69 \mathrm{E}+08$ \\
\hline 241.4791 & 113461.85 & 4.5 & (e) & 154860.81 & 4.5 & (o) & -0.78 & $1.91 \mathrm{E}+08$ \\
\hline 241.5371 & 118448.53 & 4.5 & (e) & 159837.56 & 4.5 & (o) & -0.56 & $3.14 \mathrm{E}+08$ \\
\hline 241.7628 & 113461.85 & 4.5 & (e) & 154812.25 & 3.5 & (o) & -0.11 & $8.90 \mathrm{E}+08$ \\
\hline 241.8523 & 116697.38 & 5.5 & (e) & 158032.30 & 5.5 & (o) & 0.22 & $1.92 \mathrm{E}+09$ \\
\hline 242.1330 & 119133.54 & 3.5 & (e) & 160420.64 & 4.5 & (o) & -0.54 & $3.30 \mathrm{E}+08$ \\
\hline 243.2178 & 118482.80 & 2.5 & (e) & 159585.52 & 3.5 & (o) & -0.63 & $2.62 \mathrm{E}+08$ \\
\hline
\end{tabular}


Table 3. (Continued.)

\begin{tabular}{|c|c|c|c|c|c|c|c|c|}
\hline \multirow[b]{2}{*}{ Wavelength $^{\mathrm{a}}(\mathrm{nm})$} & \multicolumn{3}{|c|}{ Lower level $^{b}$} & \multicolumn{3}{|c|}{ Upper level $^{\mathrm{b}}$} & \multirow[b]{2}{*}{$\log g f^{\mathrm{c}}$} & \multirow[b]{2}{*}{$g A^{\mathrm{c}}\left(\mathrm{s}^{-1}\right)$} \\
\hline & $\mathrm{E}\left(\mathrm{cm}^{-1}\right)$ & $J$ & Parity & $\mathrm{E}\left(\mathrm{cm}^{-1}\right)$ & $J$ & Parity & & \\
\hline 243.3890 & 110056.47 & 3.5 & (e) & 151130.44 & 4.5 & (o) & -0.64 & $2.58 \mathrm{E}+08$ \\
\hline $243.6384 *$ & 119133.54 & 3.5 & (e) & 160165.53 & 3.5 & (o) & -0.87 & $1.50 \mathrm{E}+08$ \\
\hline 244.0243 & 113118.93 & 5.5 & (e) & 154086.17 & 5.5 & (o) & -0.66 & $2.42 \mathrm{E}+08$ \\
\hline 244.2351 & 122854.37 & 3.5 & (e) & 163786.31 & 4.5 & (o) & -0.81 & $1.72 \mathrm{E}+08$ \\
\hline 246.1611 & 68803.16 & 3.5 & (o) & 109414.69 & 4.5 & (e) & -0.91 & $1.36 \mathrm{E}+08$ \\
\hline 246.1979 & 116697.38 & 5.5 & (e) & 157302.95 & 5.5 & (o) & -0.41 & $4.22 \mathrm{E}+08$ \\
\hline $246.8141 *$ & 110056.47 & 3.5 & (e) & 150560.55 & 4.5 & (o) & -0.94 & $1.27 \mathrm{E}+08$ \\
\hline 246.8617 & 110634.11 & 4.5 & (e) & 151130.44 & 4.5 & (o) & -0.58 & $2.87 \mathrm{E}+08$ \\
\hline 247.2416 & 115767.20 & 6.5 & (e) & 156201.33 & 6.5 & (o) & -0.49 & $3.52 \mathrm{E}+08$ \\
\hline $248.1421 *$ & 113118.93 & 5.5 & (e) & 153406.25 & 5.5 & (o) & -0.76 & $1.89 \mathrm{E}+08$ \\
\hline 250.0924 & 110634.11 & 4.5 & (e) & 150607.31 & 5.5 & (o) & -0.52 & $3.23 \mathrm{E}+08$ \\
\hline 250.3854 & 110634.11 & 4.5 & (e) & 150560.55 & 4.5 & (o) & -0.55 & $2.99 \mathrm{E}+08$ \\
\hline 252.5534 & 118448.53 & 4.5 & (e) & 158032.30 & 5.5 & (o) & -0.65 & $2.39 \mathrm{E}+08$ \\
\hline 252.9713 & 122515.64 & 4.5 & (e) & 162034.11 & 4.5 & (o) & -0.57 & $2.86 \mathrm{E}+08$ \\
\hline 253.2565 & 113118.93 & 5.5 & (e) & 152592.62 & 5.5 & (o) & -0.58 & $2.72 \mathrm{E}+08$ \\
\hline 253.4752 & 116697.38 & 5.5 & (e) & 156137.08 & 4.5 & (o) & -0.47 & $3.55 \mathrm{E}+08$ \\
\hline 253.8144 & 117912.91 & 3.5 & (e) & 157299.94 & 3.5 & (o) & -0.68 & $2.18 \mathrm{E}+08$ \\
\hline 255.2000 & 116697.38 & 5.5 & (e) & 155870.45 & 5.5 & (o) & -0.98 & $1.07 \mathrm{E}+08$ \\
\hline 255.4376 & 122515.64 & 4.5 & (e) & 161652.20 & 3.5 & (o) & -0.72 & $1.95 \mathrm{E}+08$ \\
\hline 255.4734 & 113461.85 & 4.5 & (e) & 152592.92 & 4.5 & (o) & -0.55 & $2.88 \mathrm{E}+08$ \\
\hline 256.2655 & 115904.51 & 1.5 & (e) & 154914.81 & 2.5 & (o) & -0.70 & $2.03 \mathrm{E}+08$ \\
\hline 258.2556 & 122515.64 & 4.5 & (e) & 161225.30 & 4.5 & (o) & -0.38 & $4.20 \mathrm{E}+08$ \\
\hline 258.4771 & 115904.51 & 1.5 & (e) & 154581.00 & 2.5 & (o) & -0.44 & $3.66 \mathrm{E}+08$ \\
\hline $259.6009 *$ & 137456.40 & 2.5 & (e) & 175965.55 & 2.5 & (o) & -0.35 & $4.44 \mathrm{E}+08$ \\
\hline 260.0858 & 116375.04 & 2.5 & (e) & 154812.25 & 3.5 & (o) & -0.87 & $1.32 \mathrm{E}+08$ \\
\hline 260.5373 & 122854.37 & 3.5 & (e) & 161225.30 & 4.5 & (o) & -0.27 & $5.31 \mathrm{E}+08$ \\
\hline 261.6621 & 116375.04 & 2.5 & (e) & 154581.00 & 2.5 & (o) & -0.43 & $3.65 \mathrm{E}+08$ \\
\hline 262.4196 & 117912.91 & 3.5 & (e) & 156008.48 & 4.5 & (o) & -0.61 & $2.39 \mathrm{E}+08$ \\
\hline 262.9998 & 113118.93 & 5.5 & (e) & 151130.44 & 4.5 & (o) & -0.98 & $1.02 \mathrm{E}+08$ \\
\hline 263.7390 & 122515.64 & 4.5 & (e) & 160420.64 & 4.5 & (o) & -0.19 & $6.21 \mathrm{E}+08$ \\
\hline 264.3027 & 118448.53 & 4.5 & (e) & 156272.69 & 3.5 & (o) & 0.39 & $2.34 \mathrm{E}+09$ \\
\hline 263.9049 & 117912.91 & 3.5 & (e) & 155794.06 & 2.5 & (o) & -0.94 & $1.12 \mathrm{E}+08$ \\
\hline 264.1562 & 117912.91 & 3.5 & (e) & 155758.06 & 2.5 & (o) & 0.12 & $1.25 \mathrm{E}+09$ \\
\hline 265.2535 & 118448.53 & 4.5 & (e) & 156137.08 & 4.5 & (o) & 0.01 & $9.71 E+08$ \\
\hline 265.5263 & 122515.64 & 4.5 & (e) & 160165.53 & 3.5 & (o) & 0.30 & $1.87 \mathrm{E}+09$ \\
\hline 265.6021 & 115767.20 & 6.5 & (e) & 153406.25 & 5.5 & (o) & 0.44 & $2.59 \mathrm{E}+09$ \\
\hline 265.6454D & 136316.05 & 6.5 & (e) & 173949.03 & 6.5 & (o) & 0.30 & $1.89 \mathrm{E}+09$ \\
\hline 265.6454D & 136316.06 & 5.5 & (e) & 173949.03 & 6.5 & (o) & -0.10 & $7.52 \mathrm{E}+08$ \\
\hline 266.1170 & 122854.37 & 3.5 & (e) & 160420.64 & 4.5 & (o) & -0.31 & $4.60 \mathrm{E}+08$ \\
\hline 266.1616 & 118448.53 & 4.5 & (e) & 156008.48 & 4.5 & (o) & -0.39 & $3.83 \mathrm{E}+08$ \\
\hline 266.6698D & 110056.47 & 3.5 & (e) & 147544.89 & 4.5 & (o) & 0.32 & $1.96 \mathrm{E}+09$ \\
\hline 266.6698D & 113118.93 & 5.5 & (e) & 150607.31 & 5.5 & (o) & 0.40 & $2.33 \mathrm{E}+09$ \\
\hline 267.0032 & 113118.93 & 5.5 & (e) & 150560.55 & 4.5 & (o) & 0.34 & $2.04 \mathrm{E}+09$ \\
\hline 267.3797 & 116697.38 & 5.5 & (e) & 154086.17 & 5.5 & (o) & -0.86 & $1.28 \mathrm{E}+08$ \\
\hline $267.6769 *$ & 137456.40 & 2.5 & (e) & 174803.78 & 2.5 & (o) & -0.99 & $9.50 \mathrm{E}+07$ \\
\hline 267.8586 & 122515.64 & 4.5 & (e) & 159837.56 & 4.5 & (o) & -0.37 & $3.98 \mathrm{E}+08$ \\
\hline 267.9366 & 122854.37 & 3.5 & (e) & 160165.53 & 3.5 & (o) & -0.22 & $5.62 \mathrm{E}+08$ \\
\hline 268.1946 & 118482.80 & 2.5 & (e) & 155758.06 & 2.5 & (o) & -0.61 & $2.28 \mathrm{E}+08$ \\
\hline 269.1319 & 113461.85 & 4.5 & (e) & 150607.31 & 5.5 & (o) & 0.30 & $1.83 \mathrm{E}+09$ \\
\hline 269.1777 & 119133.54 & 3.5 & (e) & 156272.69 & 3.5 & (o) & -0.20 & $5.85 \mathrm{E}+08$ \\
\hline $269.2461 *$ & 137456.40 & 1.5 & (e) & 174586.12 & 1.5 & (o) & 0.003 & $9.26 \mathrm{E}+08$ \\
\hline 269.6809 & 122515.64 & 4.5 & (e) & 159585.52 & 3.5 & (o) & -0.54 & $2.64 \mathrm{E}+08$ \\
\hline 270.1642 & 119133.54 & 3.5 & (e) & 156137.08 & 4.5 & (o) & 0.11 & $1.18 \mathrm{E}+09$ \\
\hline 270.1759 & 117912.91 & 3.5 & (e) & 154914.81 & 2.5 & (o) & -0.49 & $2.98 \mathrm{E}+08$ \\
\hline 270.3133 & 122854.37 & 3.5 & (e) & 159837.56 & 4.5 & (o) & -0.02 & $8.65 \mathrm{E}+08$ \\
\hline 270.8434 & 110634.11 & 4.5 & (e) & 147544.89 & 4.5 & (o) & 0.27 & $1.70 \mathrm{E}+09$ \\
\hline 270.9271D & 117912.91 & 3.5 & (e) & 154812.25 & 3.5 & (o) & -0.15 & $6.42 \mathrm{E}+08$ \\
\hline 270.9271D & 137456.41 & 2.5 & (e) & 174355.70 & 1.5 & (o) & -0.80 & $1.44 \mathrm{E}+08$ \\
\hline 271.1061 & 119133.54 & 3.5 & (e) & 156008.48 & 4.5 & (o) & -0.41 & $3.51 \mathrm{E}+08$ \\
\hline 271.6162 & 115904.51 & 1.5 & (e) & 152710.30 & 2.5 & (o) & -0.09 & $7.39 \mathrm{E}+08$ \\
\hline 272.1686 & 122854.37 & 3.5 & (e) & 159585.52 & 3.5 & (o) & -0.94 & $1.03 \mathrm{E}+08$ \\
\hline 272.3338 & 116697.38 & 5.5 & (e) & 153406.25 & 5.5 & (o) & 0.22 & $1.48 \mathrm{E}+09$ \\
\hline $272.3512 \mathrm{D}$ & 136316.05 & 6.5 & (e) & 173022.52 & 5.5 & (o) & 0.21 & $1.46 \mathrm{E}+09$ \\
\hline $272.3512 \mathrm{D}$ & 136316.06 & 5.5 & (e) & 173022.52 & 5.5 & (o) & 0.39 & $2.23 \mathrm{E}+09$ \\
\hline
\end{tabular}


Table 3. (Continued.)

\begin{tabular}{|c|c|c|c|c|c|c|c|c|}
\hline \multirow[b]{2}{*}{ Wavelength $^{\mathrm{a}}(\mathrm{nm})$} & \multicolumn{3}{|c|}{ Lower level $^{\mathrm{b}}$} & \multicolumn{3}{|c|}{ Upper level $^{\mathrm{b}}$} & \multirow[b]{2}{*}{$\log g f^{\mathrm{c}}$} & \multirow[b]{2}{*}{$g A^{\mathrm{c}}\left(\mathrm{s}^{-1}\right)$} \\
\hline & $\mathrm{E}\left(\mathrm{cm}^{-1}\right)$ & $J$ & Parity & $\mathrm{E}\left(\mathrm{cm}^{-1}\right)$ & $J$ & Parity & & \\
\hline $272.9603 *$ & 119133.54 & 3.5 & (e) & 155758.06 & 2.5 & (o) & -0.77 & $1.50 \mathrm{E}+08$ \\
\hline 273.1597D & 136316.05 & 6.5 & (e) & 172913.80 & 6.5 & (o) & 0.18 & $1.36 \mathrm{E}+09$ \\
\hline 273.1597D & 136316.06 & 5.5 & (e) & 172913.80 & 6.5 & (o) & -0.24 & $5.13 \mathrm{E}+08$ \\
\hline 274.4035 & 118482.80 & 2.5 & (e) & 154914.81 & 2.5 & (o) & -0.81 & $1.39 \mathrm{E}+08$ \\
\hline 274.5511 & 118448.53 & 4.5 & (e) & 154860.81 & 4.5 & (o) & -0.87 & $1.20 \mathrm{E}+08$ \\
\hline 275.1333 & 116375.04 & 2.5 & (e) & 152710.30 & 2.5 & (o) & -0.31 & $4.34 \mathrm{E}+08$ \\
\hline 275.1775 & 118482.80 & 2.5 & (e) & 154812.25 & 3.5 & (o) & 0.06 & $1.02 \mathrm{E}+09$ \\
\hline
\end{tabular}

${ }^{\text {a }}$ Vacuum (below $200 \mathrm{~nm}$ ) and air (above $200 \mathrm{~nm}$ ) wavelengths from Wyart et al (2007); D : doubly classified line; $\mathrm{T}$ : triply classified line; * deduced from available experimental energy level values.

${ }^{\mathrm{b}}$ Experimental energy levels from Wyart et al (2007).

${ }^{c}$ This work.

strongly interact with lower configurations. More precisely, in their analysis of the Nd IV spectrum, Wyart et al (2007) showed that, owing to configuration mixing between $5 p^{6} 4 f^{2} 6 p$ and the core-excited $5 p^{5} 4 f^{4}$, numerous $5 p^{6} 4 f^{2} 5 d-$ $5 \mathrm{p}^{5} 4 \mathrm{f}^{4}$ transitions, normally dipole-forbidden, were observed experimentally. They also showed that the addition of the $5 p^{5} 4 f^{3} 5 d$ configuration (still unknown) to $5 p^{6} 4 f^{2}(5 d+6 s)$ in a three-configuration basis improved significantly the calculated energies in $4 \mathrm{f}^{2} 5 \mathrm{~d}$ and $4 \mathrm{f}^{2} 6 \mathrm{~s}$ configurations and reduced the transition probabilities for $5 p^{6} 4 f^{3}-5 p^{6} 4 f^{2} 5 d$ by a factor of about 2 . To give an idea, the increasing influence of $5 p^{5} 4 f^{4}$ along the lanthanum isoelectronic sequence is illustrated on figure 1 in which calculated HFR average energies corresponding to odd-parity configurations are plotted for La I, Ce II, Pr III and Nd IV. When looking at this figure, it is clear that the $5 \mathrm{p}^{5} 4 \mathrm{f}^{4}$ configuration must play a much more important role in $\mathrm{Nd}$ IV than in the previous ions of the sequence for the description of lower configurations. Consequently, it is obvious that a core-polarization model potential is not sufficient to take such core-valence interactions into account which can only be considered by the explicit inclusion of core-excited configurations in the multiconfiguration expansions. The following HFR model was thus adopted for Nd IV : $4 \mathrm{f}^{3}+$ $4 f^{2} 6 p+4 f^{2} 5 f+4 f^{2} 6 f+4 f 5 d^{2}+4 f 6 s^{2}+4 f 6 p^{2}+4 f 6 d^{2}+$ $4 f 5 d 6 s+4 f 5 d 6 d+5 d 6 s 6 p+5 d^{2} 6 p+6 s^{2} 6 p+6 p^{3}+5 p^{5} 4 f^{4}$ for the odd parity and $4 \mathrm{f}^{2} 5 \mathrm{~d}+4 \mathrm{f}^{2} 6 \mathrm{~s}+4 \mathrm{f}^{2} 6 \mathrm{~d}+4 \mathrm{f}^{2} 5 \mathrm{~g}+4 \mathrm{f} 5 \mathrm{~d} 6 \mathrm{p}$ $+4 \mathrm{f} 5 \mathrm{~d} 5 \mathrm{f}+4 \mathrm{f} 6 \mathrm{~s} 6 \mathrm{p}+5 \mathrm{~d}^{3}+5 \mathrm{~d}^{2} 6 \mathrm{~s}+5 \mathrm{~d}^{2} 6 \mathrm{~d}+5 \mathrm{~d} 6 \mathrm{~s}^{2}+5 \mathrm{~d} 6 \mathrm{p}^{2}+$ $6 s 6 p^{2}+5 p^{5} 4 f^{3} 5 d$ for the even parity.

In addition, average energies, $E_{\mathrm{av}}$, Slater parameters, $F^{k}$ and $G^{k}$, spin-orbit integrals, $\zeta_{n l}$ and effective interaction parameters were adjusted with a least-squares optimization program minimizing the discrepancies between the calculated and the experimental energy levels reported by Wyart et al (2007) for $4 f^{3}, 4 f^{2} 6 p, 5 p^{5} 4 f^{4}$ odd-parity configurations and $4 f^{2} 5 d, 4 f^{2} 6 s$ even-parity configurations. For electrostatic interaction Slater parameters $\left(\mathrm{F}^{k}, \mathrm{G}^{k}, \mathrm{R}^{k}\right)$ not adjusted in the fitting process, a scaling factor of 0.85 was applied to the HFR $a b$ initio values, as recommended by Cowan (1981). The standard deviations of the fits were found to be equal to $89 \mathrm{~cm}^{-1}$ and $115 \mathrm{~cm}^{-1}$ in the odd parity (111 levels fitted with 29 adjustable parameters) and even parity (121 levels fitted with 21 parameters), respectively. Details about the final radial

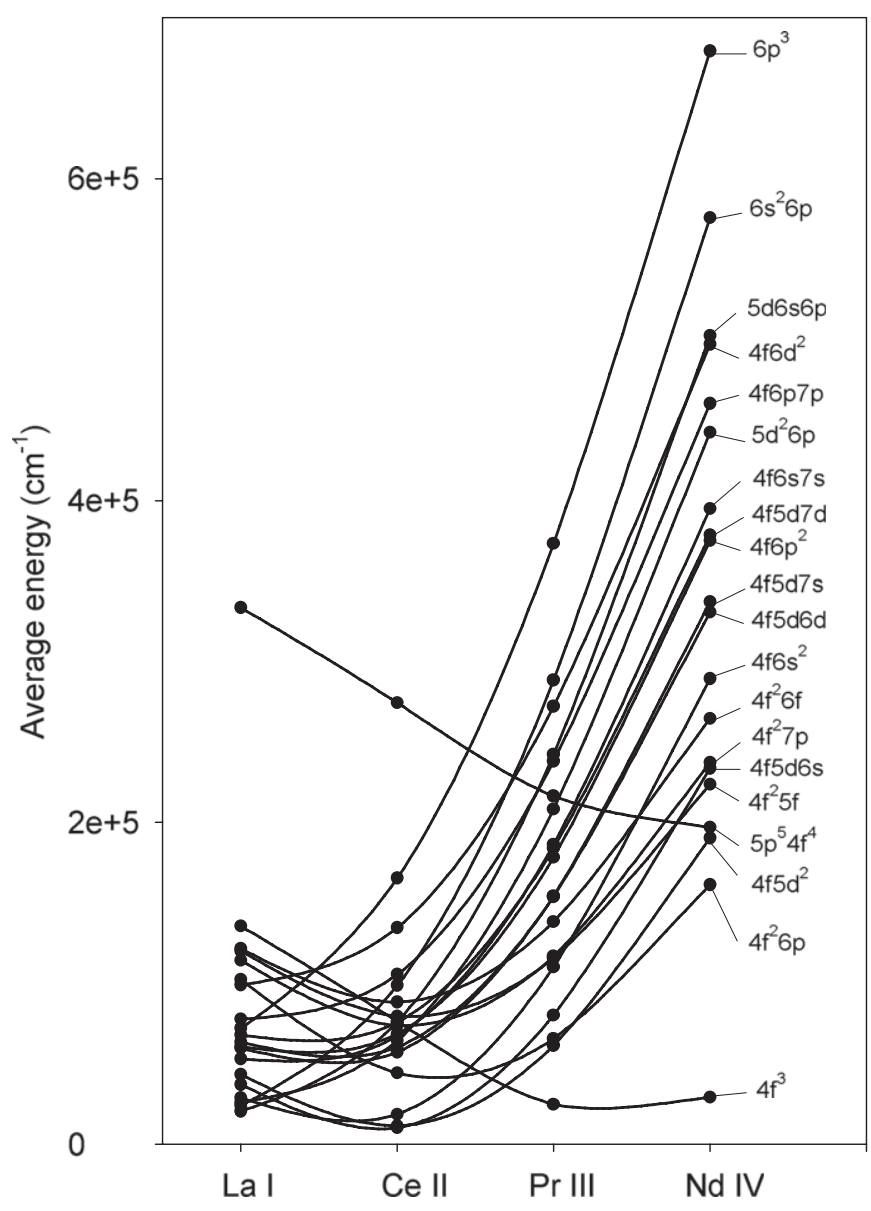

Figure 1. Computed average energies for odd-parity configurations along the lanthanum isoelectronic sequence from La I to Nd IV.

parameters adopted in our calculations are given in tables 1 and 2.

\section{Results and discussion}

\subsection{Electric dipole transitions}

Radiative parameters (oscillator strengths and transition probabilities) computed in this work are reported in table 3 for Nd IV electric dipole lines between 106.8 and $275.2 \mathrm{~nm}$. 


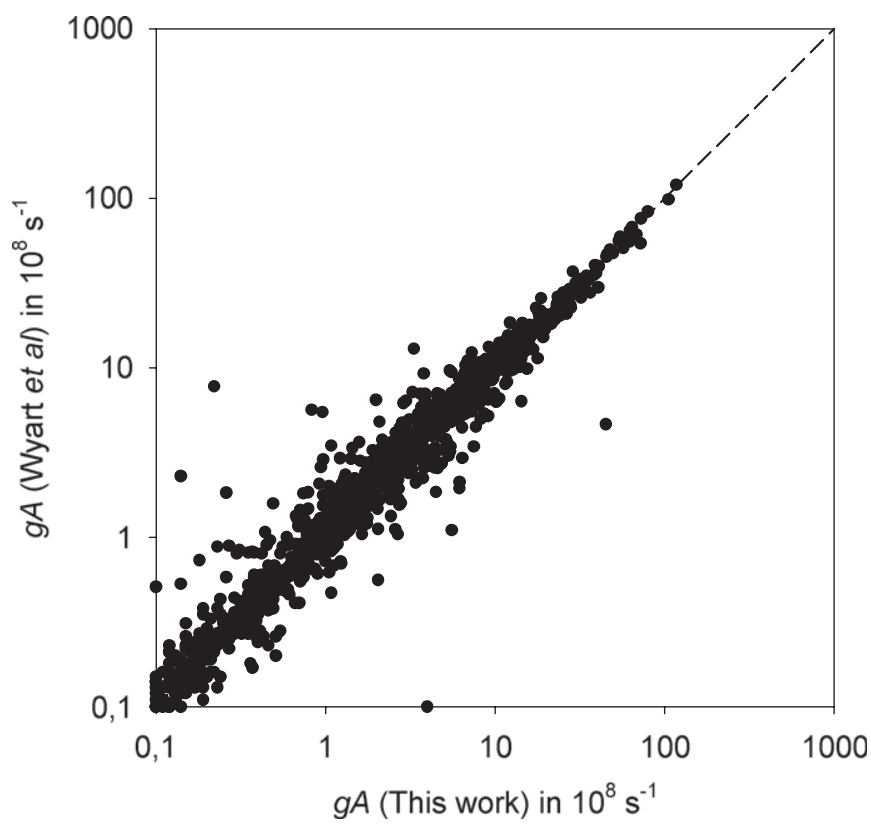

Figure 2. Comparison between calculated radiative transition probabilities as obtained in the present work and those published by Wyart et al (2007) for allowed electric dipole lines in Nd IV.

Table 4. Comparison of computed radiative lifetimes for some levels belonging to the $4 \mathrm{f}^{2} 5 \mathrm{~d}$ configuration of $\mathrm{Nd}$ IV.

\begin{tabular}{lcccc}
\hline & & \multicolumn{3}{c}{ Lifetime (ns) } \\
\cline { 3 - 5 } Level $^{\mathrm{a}}$ & $\mathrm{E}\left(\mathrm{cm}^{-1}\right)^{\mathrm{a}}$ & This work & Dzuba et al ${ }^{\mathrm{b}}$ & ${\text { Wyart } \text { et } a l^{\text {c }}}$ \\
\hline$\left({ }^{3} \mathrm{H}\right)^{2} \mathrm{H}_{9 / 2}$ & 70817.12 & 16 & 6.6 & 14.5 \\
$\left({ }^{3} \mathrm{H}\right)^{4} \mathrm{~K}_{11 / 2}$ & 71744.57 & 134 & 68.5 & 116 \\
$\left({ }^{3} \mathrm{H}\right)^{4} \mathrm{I}_{9 / 2}$ & 73366.17 & 21.4 & 9.4 & 16.9 \\
$\left({ }^{3} \mathrm{H}\right)^{4} \mathrm{I}_{11 / 2}$ & 73556.74 & 16.1 & 17.5 & 16.6 \\
$\left({ }^{3} \mathrm{H}\right)^{4} \mathrm{G}_{5 / 2}$ & 73616.22 & 31.8 & 23.7 & 29.9 \\
$\left({ }^{3} \mathrm{H}\right)^{4} \mathrm{I}_{11 / 2}$ & 74571.83 & 25.4 & 6.4 & 17.0 \\
$\left({ }^{3} \mathrm{H}\right)^{4} \mathrm{~K}_{13 / 2}$ & 74673.95 & 157 & 75.2 & 133 \\
$\left({ }^{3} \mathrm{H}\right)^{4} \mathrm{G}_{7 / 2}$ & 75666.91 & 24.3 & 15.4 & 21.2 \\
$\left({ }^{3} \mathrm{H}\right)^{4} \mathrm{I}_{13 / 2}$ & 76471.40 & 13.2 & 5.5 & 10.8 \\
$\left({ }^{3} \mathrm{H}\right)^{4} \mathrm{~K}_{15 / 2}$ & 77539.71 & 159 & 79.2 & 134 \\
$\left({ }^{3} \mathrm{H}\right)^{4} \mathrm{G}_{9 / 2}$ & 77809.61 & 23.1 & 10.8 & 20.1 \\
$\left({ }^{3} \mathrm{H}\right)^{4} \mathrm{I}_{15 / 2}$ & 78864.24 & 13.0 & 5.4 & 10.7 \\
$\left({ }^{3} \mathrm{H}\right)^{4} \mathrm{G}_{11 / 2}$ & 80080.41 & 26.5 & 13.0 & 23.7 \\
$\left({ }^{3} \mathrm{H}\right)^{4} \mathrm{~K}_{17 / 2}$ & 80431.49 & 156 & 77.5 & 134 \\
\hline
\end{tabular}

${ }^{\text {a }}$ From Wyart et al (2007).

${ }^{\mathrm{b}}$ Calculations from Dzuba et al (2003).

${ }^{c}$ Calculations from Wyart et al (2007).

The results presented in this table are limited to transitions for which $\log g f$ is greater than -1.0 .

To our knowledge, prior to our work, transition probabilities in Nd IV were only published by Wyart et al (2007) who also used the HFR method combined with parametric least-squares fits but including a rather limited set of interacting configurations, i.e. $5 p^{6} 4 f 5 d^{2}+5 p^{6} 4 f 5 d 6 s+$ $5 \mathrm{p}^{6} 4 \mathrm{f}^{6} \mathrm{~s}^{2}+5 \mathrm{p}^{6} 4 \mathrm{f}^{3}+5 \mathrm{p}^{6} 4 \mathrm{f}^{2} 6 \mathrm{p}+5 \mathrm{p}^{5} 4 \mathrm{f}^{4}$ for the odd parity and $5 p^{6} 4 f^{2} 5 d+5 p^{6} 4 f^{2} 6 s+5 p^{5} 4 f^{3} 5 d$ for the even parity. The comparison between Wyart et al's results and our $g A$-values is illustrated in figure 2, showing a general good agreement (within 25\%) between both sets of data.
Table 5. Transition probabilities for forbidden lines within the $4 \mathrm{f}^{3}$ ground-state configuration of Nd IV. Only transitions with $g A \geqslant 0.1 \mathrm{~s}^{-1}$ are listed in the table.

\begin{tabular}{|c|c|c|c|c|}
\hline $\begin{array}{l}\text { Wavelength } \\
(\mathrm{nm})\end{array}$ & Transition & Type $^{\text {b }}$ & $\begin{array}{l}g A \\
\text { (This work) } \\
\left(\mathrm{s}^{-1}\right)\end{array}$ & $\begin{array}{l}g A \\
(\text { Other })^{\mathrm{c}} \\
\left(\mathrm{s}^{-1}\right)\end{array}$ \\
\hline 175.0323 & ${ }^{2} \mathrm{H}_{9 / 2}^{\circ} 2{ }^{2} \mathrm{~F}_{5 / 2}^{\circ} 1$ & E2 & $5.36 \mathrm{E}+00$ & \\
\hline 178.1258 & ${ }^{4} S_{3 / 2}^{\circ}-{ }^{\circ} F_{5 / 2}^{\circ} 1$ & E2 & $2.24 \mathrm{E}-01$ & \\
\hline 181.7829 & ${ }^{4} \mathrm{~S}_{3 / 2}^{\circ}-{ }^{2} \mathrm{~F}_{7 / 2}^{\circ} 1$ & E2 & $1.05 \mathrm{E}+00$ & \\
\hline 185.8450 & ${ }^{4} \mathrm{~F}_{9 / 2}^{\circ}-{ }^{2} \mathrm{~F}_{7 / 2}^{\circ} 1$ & $\mathrm{M} 1+\mathrm{E} 2$ & $9.34 \mathrm{E}-01$ & \\
\hline 189.9637 & ${ }^{2} \mathrm{H}_{11 / 2}^{\circ} 2{ }^{2} \mathrm{~F}_{7 / 2}^{\circ} 1$ & E2 & $6.14 \mathrm{E}+00$ & \\
\hline 191.2870 & ${ }^{2} \mathrm{G}_{7 / 2}^{\circ} 2{ }^{2}{ }^{2} \mathrm{~F}^{\circ 5 / 2} 1$ & $\mathrm{M} 1+\mathrm{E} 2$ & $5.15 \mathrm{E}+00$ & \\
\hline 198.4450 & ${ }^{4} \mathrm{G}_{7 / 2}^{\circ}-{ }^{2} \mathrm{~F}_{5 / 2}^{\circ} 1$ & $\mathrm{M} 1+\mathrm{E} 2$ & $4.10 \mathrm{E}+00$ & \\
\hline 202.9294 & ${ }^{4} \mathrm{G}_{7 / 2}^{\circ}-{ }^{\circ} \mathrm{F}_{7 / 2}^{\circ} 1$ & E2 & $4.19 \mathrm{E}-01$ & \\
\hline 204.7124 & ${ }^{4} \mathrm{G}_{9 / 2}^{\circ}-{ }^{\circ} \mathrm{F}_{7 / 2}^{\circ} 1$ & $\mathrm{M} 1+\mathrm{E} 2$ & $4.61 \mathrm{E}+00$ & \\
\hline 207.2664 & ${ }^{2} \mathrm{D}_{3 / 2}^{\circ} 1-^{2} \mathrm{~F}_{5 / 2}^{\circ} 1$ & $\mathrm{M} 1+\mathrm{E} 2$ & $2.65 \mathrm{E}+00$ & \\
\hline 211.3059 & ${ }^{2} \mathrm{G}_{9 / 2}^{\circ} 2-{ }^{2} \mathrm{~F}_{7 / 2}^{\circ} 1$ & $\mathrm{M} 1+\mathrm{E} 2$ & $6.63 \mathrm{E}+00$ & \\
\hline 212.2369 & ${ }^{2} \mathrm{D}_{3 / 2}^{\circ} 1-{ }^{2} \mathrm{~F}_{7 / 2}^{\circ} 1$ & E2 & $5.92 \mathrm{E}+00$ & \\
\hline 216.6465 & ${ }^{2} \mathrm{P}_{1 / 2}^{\circ}-{ }^{\circ} \mathrm{F}_{5 / 2}^{\circ} 1$ & E2 & $8.87 \mathrm{E}+00$ & \\
\hline 224.8007 & ${ }^{2} \mathrm{D}_{5 / 2}^{\circ} 1-{ }^{2} \mathrm{~F}_{7 / 2}^{\circ} 1$ & M1 & $1.35 \mathrm{E}+00$ & \\
\hline 231.5637 & ${ }^{2} \mathrm{P}_{3 / 2}^{\circ}-{ }^{\circ} \mathrm{F}_{5 / 2}^{\circ} 1$ & M1 & $6.33 \mathrm{E}-01$ & \\
\hline 237.7850 & ${ }^{2} \mathrm{P}_{3 / 2}^{\circ}-{ }^{2} \mathrm{~F}_{7 / 2}^{\circ} 1$ & E2 & $7.32 \mathrm{E}+00$ & \\
\hline 244.2923 & ${ }^{4} \mathrm{D}_{3 / 2}^{\circ}-{ }^{2} \mathrm{~F}_{5 / 2}^{\circ} 1$ & $\mathrm{M} 1+\mathrm{E} 2$ & $3.85 \mathrm{E}+00$ & \\
\hline 245.3746 & ${ }^{4} \mathrm{D}_{5 / 2}^{\circ}-{ }^{\circ} \mathrm{F}_{5 / 2}^{\circ} 1$ & E2 & $3.56 \mathrm{E}-01$ & \\
\hline 247.4979 & ${ }^{4} \mathrm{D}_{1 / 2}^{\circ}-{ }^{\circ} \mathrm{F}_{5 / 2}^{\circ} 1$ & E2 & $2.84 \mathrm{E}-01$ & \\
\hline 251.2265 & ${ }^{4} \mathrm{D}_{3 / 2}^{\circ}-{ }^{2} \mathrm{~F}_{7 / 2}^{\circ} 1$ & E2 & $2.78 \mathrm{E}-01$ & \\
\hline 252.3712 & ${ }^{4} \mathrm{D}_{5 / 2}^{\circ}-{ }^{\circ} \mathrm{F}_{3.5}^{\circ}{ }_{1}^{\circ}$ & $\mathrm{M} 1+\mathrm{E} 2$ & $5.05 \mathrm{E}+00$ & \\
\hline 258.8341 & ${ }^{2} \mathrm{I}_{11 / 2}^{\circ}-{ }^{2} \mathrm{~F}_{7 / 2}^{\circ} 1$ & E2 & $1.76 \mathrm{E}+00$ & \\
\hline 267.2073 & ${ }^{4} \mathrm{~F}_{5 / 2}^{\circ}-{ }^{2} \mathrm{G}_{7 / 2}^{\circ} 1$ & M1 & $1.06 \mathrm{E}+00$ & \\
\hline 276.2268 & ${ }^{2} \mathrm{H}_{9 / 2}^{\circ} 1{ }^{2} \mathrm{~F}_{5 / 2}^{\circ} 1$ & E2 & $1.10 \mathrm{E}+01$ & \\
\hline 280.3642 & ${ }^{2} \mathrm{D}_{3 / 2}^{\circ} 2-{ }^{2} \mathrm{~F}_{5 / 2}^{\circ} 1$ & $\mathrm{M} 1+\mathrm{E} 2$ & $2.88 \mathrm{E}+00$ & \\
\hline 285.1252 & ${ }^{2} \mathrm{H}_{9 / 2}^{\circ} 1-{ }^{2} \mathrm{~F}_{7 / 2}^{\circ} 1$ & $\mathrm{M} 1+\mathrm{E} 2$ & $1.10 \mathrm{E}+00$ & \\
\hline 287.9450 & ${ }^{2} \mathrm{D}_{5 / 2}^{\circ} 2-{ }^{2} \mathrm{~F}_{5 / 2}^{\circ} 1$ & E2 & $1.10 \mathrm{E}+00$ & \\
\hline 296.9440 & ${ }^{2} \mathrm{H}_{11 / 2}^{\circ} 1-{ }^{2} \mathrm{~F}_{3.5}^{\circ} 1$ & E2 & $1.02 \mathrm{E}+01$ & \\
\hline 297.6275 & ${ }^{2} \mathrm{D}_{5 / 2}^{\circ} 2-{ }^{2} \mathrm{~F}_{7 / 2}^{\circ} 1$ & E2 & $1.25 \mathrm{E}+00$ & \\
\hline 302.8419 & ${ }^{2} \mathrm{H}_{11 / 2}^{\circ} 2{ }^{\circ} \mathrm{G}_{9 / 2}^{\circ} 1$ & $\mathrm{M} 1+\mathrm{E} 2$ & $7.78 \mathrm{E}-01$ & \\
\hline 308.0410 & ${ }^{4} \mathrm{G}_{5 / 2}^{\circ}-{ }^{2} \mathrm{G}_{7 / 2}^{\circ} 1$ & $\mathrm{M} 1+\mathrm{E} 2$ & $4.12 \mathrm{E}+00$ & \\
\hline 320.1208 & ${ }^{4} \mathrm{I}_{13 / 2}^{\circ}-{ }^{2} \mathrm{H}_{11 / 2}^{\circ} 1$ & $\mathrm{M} 1+\mathrm{E} 2$ & $9.64 \mathrm{E}-01$ & $2.89 \mathrm{E}+00$ \\
\hline 331.2507 & ${ }^{4} \mathbf{I}_{9 / 2}^{\circ}-{ }^{2} \mathrm{I}_{11 / 2}^{\circ}$ & $\mathrm{M} 1+\mathrm{E} 2$ & $2.93 E+00$ & $2.62 \mathrm{E}+01$ \\
\hline 341.9607 & ${ }^{2} \mathrm{~F}_{5 / 2}^{\circ} 2-{ }^{2} \mathrm{~F}_{7 / 2}^{\circ} 1$ & E2 & $7.61 \mathrm{E}-01$ & \\
\hline 345.6838 & ${ }^{2} \mathrm{~F}_{7 / 2}^{\mathrm{o}} 2-{ }^{2} \mathrm{~F}_{5 / 2}^{\mathrm{o}} 1$ & E2 & $2.71 \mathrm{E}-01$ & \\
\hline 351.2693 & ${ }^{2} \mathrm{D}_{3 / 2}^{\circ} 1-{ }^{2} \mathrm{G}_{7 / 2}^{\circ} 1$ & E2 & $2.92 \mathrm{E}-01$ & \\
\hline 353.6964 & ${ }^{4} \mathrm{~F}_{5 / 2}^{\circ}-{ }^{\circ} \mathrm{F}_{7 / 2}^{\circ} 2$ & $\mathrm{M} 1+\mathrm{E} 2$ & $3.30 \mathrm{E}+00$ & \\
\hline 354.3527 & ${ }^{2} \mathrm{H}_{9 / 2}^{\circ} 2-{ }^{2} \mathrm{~F}_{7 / 2}^{\circ} 2$ & M1 & $1.14 \mathrm{E}+00$ & \\
\hline 358.7073 & ${ }^{4} \mathrm{~F}_{3 / 2}^{\circ}-{ }^{2} \mathrm{~F}_{5 / 2}^{\circ} 2$ & $\mathrm{M} 1+\mathrm{E} 2$ & $5.01 \mathrm{E}+00$ & $9.61 \mathrm{E}+00$ \\
\hline 359.7331 & ${ }^{2} \mathrm{~F}_{7 / 2}^{\circ} 2-{ }^{2} \mathrm{~F}_{7 / 2}^{\circ} 1$ & E2 & $7.30 \mathrm{E}-01$ & \\
\hline 368.5102 & ${ }^{4} \mathrm{I}_{13 / 2}^{\mathrm{o}}-{ }^{2} \mathrm{~L}_{15 / 2}^{\circ}$ & $\mathrm{M} 1+\mathrm{E} 2$ & $4.86 \mathrm{E}-01$ & $4.79 \mathrm{E}-01$ \\
\hline 368.5579 & ${ }^{4} \mathrm{G}_{11 / 2}^{\circ}-{ }^{2} \mathrm{G}_{9 / 2}^{\circ} 1$ & $\mathrm{M} 1+\mathrm{E} 2$ & $5.60 \mathrm{E}+00$ & \\
\hline 373.4724 & ${ }^{2} \mathrm{H}_{9 / 2}^{\circ} 2-{ }^{2} \mathrm{~F}_{5 / 2}^{\circ} 2$ & E2 & $9.71 \mathrm{E}-01$ & $3.6 \mathrm{E}-01$ \\
\hline 376.1858 & ${ }^{4} \mathrm{I}_{15 / 2}^{\circ}-{ }^{2} \mathrm{~L}_{17 / 2}^{\circ}$ & $\mathrm{M} 1+\mathrm{E} 2$ & $2.78 \mathrm{E}-01$ & $2.93 \mathrm{E}-01$ \\
\hline 380.5181 & ${ }^{4} \mathbf{I}_{13 / 2}^{\circ}-{ }^{2} I_{11 / 2}^{\circ}$ & M1 & $4.23 \mathrm{E}-01$ & $4.68 \mathrm{E}+00$ \\
\hline 384.2428 & ${ }^{4} \mathrm{~F}_{9 / 2}^{\circ}-{ }^{2} \mathrm{~F}_{7 / 2}^{\circ} 2$ & M1 & $8.99 \mathrm{E}+00$ & \\
\hline 386.7585 & ${ }^{4} \mathrm{~F}_{7 / 2}^{\circ}-{ }^{2} \mathrm{~F}_{5 / 2}^{o} 2$ & $\mathrm{M} 1+\mathrm{E} 2$ & $1.42 \mathrm{E}+00$ & $2.31 \mathrm{E}+00$ \\
\hline 390.6007 & ${ }^{4} \mathbf{I}_{15 / 2}^{\mathrm{o}}-{ }^{2} \mathbf{I}_{13 / 2}^{\mathrm{o}}$ & M1 & $4.37 \mathrm{E}+00$ & $2.55 \mathrm{E}+01$ \\
\hline 402.2818 & ${ }^{2} \mathrm{H}_{11 / 2}^{\circ} 2-{ }^{\circ} \mathrm{F}_{7 / 2}^{\circ} 2$ & E2 & $1.82 \mathrm{E}+00$ & \\
\hline 402.4733 & ${ }^{2} \mathrm{D}_{5 / 2}^{\circ} 1-{ }^{2} \mathrm{G}_{9 / 2}^{\circ} 1$ & E2 & $5.62 \mathrm{E}-01$ & \\
\hline 406.8267 & ${ }^{4} \mathrm{~F}_{9 / 2}^{\circ}-{ }^{\circ} \mathrm{F}_{5 / 2}^{\circ} 2$ & E2 & $3.07 \mathrm{E}-01$ & $1.03 \mathrm{E}-01$ \\
\hline 425.1330 & ${ }^{4} \mathrm{~F}_{3 / 2}^{\circ}-{ }^{2} \mathrm{D}_{5 / 2}^{\circ} 2$ & M1 & $1.39 \mathrm{E}+00$ & $2.30 \mathrm{E}+00$ \\
\hline 444.9926 & ${ }^{4} \mathrm{~F}_{5 / 2}^{\circ}-{ }^{2} \mathrm{D}_{5 / 2}^{\circ} 2$ & $\mathrm{M} 1+\mathrm{E} 2$ & $5.17 \mathrm{E}-01$ & $5.97 \mathrm{E}-01$ \\
\hline 446.0319 & ${ }^{2} \mathrm{H}_{9 / 2}^{\circ} 2-{ }^{2} \mathrm{D}_{5 / 2}^{\circ} 2$ & E2 & $2.45 \mathrm{E}-01$ & \\
\hline 456.2158 & ${ }^{2} \mathrm{G}_{7 / 2}^{\circ} 2-{ }^{2} \mathrm{~F}_{5 / 2}^{\circ} 2$ & $\mathrm{M} 1+\mathrm{E} 2$ & $1.23 \mathrm{E}+01$ & $1.22 \mathrm{E}+01$ \\
\hline
\end{tabular}


Table 5. (Continued.)

\begin{tabular}{|c|c|c|c|c|}
\hline $\begin{array}{l}\text { Javelengt } \\
\mathrm{im})\end{array}$ & Trans & Type $^{\text {b }}$ & $\begin{array}{l}g A \\
\text { (This work) } \\
\left(\mathrm{s}^{-1}\right)\end{array}$ & $\begin{array}{l}g A \\
(\text { Other })^{\mathrm{c}} \\
\left(\mathrm{s}^{-1}\right)\end{array}$ \\
\hline 7.30222 & & M1 & $77 \mathrm{E}+00$ & +00 \\
\hline 54.3970 & ${ }^{4} \mathrm{~F}_{5 / 2}^{\mathrm{O} / 2}$ & $\mathrm{M} 1+\mathrm{E} 2$ & $7.24 \mathrm{E}-01$ & $7.24 \mathrm{E}-01$ \\
\hline 65.1137 & ${ }^{4} \mathrm{~F}_{7 / 2}^{\circ}-{ }^{\circ} \mathrm{D}_{5 / 2}^{\circ} 2$ & $\mathrm{M} 1+\mathrm{E} 2$ & $6.91 \mathrm{E}-01$ & $5.97 \mathrm{E}-01$ \\
\hline 75.0866 & ${ }^{4} \mathrm{G}_{9 / 2}^{\circ}-{ }^{2} \mathrm{I}$ & M1 & $1.28 \mathrm{E}+01$ & \\
\hline 76.7375 & ${ }^{4} \mathrm{D}_{5 / 2}^{\circ}$ & M1 & $1.39 \mathrm{E}-01$ & \\
\hline 81.5577 & ${ }^{2} \mathrm{G}_{9 / 2}^{\circ}$ & E2 & $1.52 \mathrm{E}-01$ & \\
\hline 99.1712 & ${ }^{4} \mathrm{G}_{7 / 2}^{\circ}-{ }^{2} \mathrm{~F}_{5 / 2}^{\circ} 2$ & $\mathrm{M} 1+\mathrm{E} 2$ & $4.01 \mathrm{E}+00$ & $4.23 \mathrm{E}+00$ \\
\hline 00.3354 & ${ }^{2} \mathrm{I}_{11 / 2}^{\circ}$ & E2 & $7.96 \mathrm{E}-01$ & \\
\hline 40 & ${ }^{2} \mathrm{G}_{7 / 2}^{\circ} 1-{ }^{2} \mathrm{~F}_{7 / 2}^{\circ} 1$ & $\mathrm{M} 1+\mathrm{E} 2$ & $7.22 \mathrm{E}+00$ & \\
\hline 9.2640 & $\mathrm{~F}_{7 / 2}^{\circ} 1$ & $\mathrm{M} 1+\mathrm{E} 2$ & $8.22 \mathrm{E}+00$ & \\
\hline 10.1 & ${ }^{4} I_{11 / 2}^{\circ}$ & $\mathrm{M} 1+\mathrm{E} 2$ & $3.55 \mathrm{E}-01$ & $2.79 \mathrm{E}-01$ \\
\hline 12.1705 & ${ }^{2} \mathrm{~F}_{7 / 2}^{\circ} 2$ & $\mathrm{M} 1+\mathrm{E} 2$ & $6.07 \mathrm{E}+00$ & \\
\hline 66 & ${ }^{2} \mathrm{G}_{9 / 2}^{\circ} 1$ & $\mathrm{M} 1+\mathrm{E} 2$ & $1.22 \mathrm{E}+00$ & \\
\hline 36. & $\mathrm{~F}_{7 / 2}^{\circ} 1$ & $\mathrm{M} 1+\mathrm{E} 2$ & $1.13 \mathrm{E}+01$ & \\
\hline 9 & & $\mathrm{M} 1+\mathrm{E} 2$ & $1.17 \mathrm{E}-01$ & $1.86 \mathrm{E}-01$ \\
\hline 51.1160 & ${ }^{4} \mathrm{I}_{1}^{\circ}$ & $\mathrm{M} 1+\mathrm{E} 2$ & $1.10 \mathrm{E}+00$ & $1.28 \mathrm{E}+00$ \\
\hline 60 & ${ }^{4} \mathbf{I}_{13 / 2}^{\circ}-$ & M1 & $1.63 \mathrm{E}+01$ & $.56 \mathrm{E}+01$ \\
\hline 4 & ${ }^{4} \mathbf{I}_{11}^{\circ}$ & M1 & $1.53 \mathrm{E}+01$ & $74 \mathrm{E}+01$ \\
\hline 77 & & $\mathrm{M} 1+\mathrm{E} 2$ & $1.68 \mathrm{E}+00$ & $1.70 \mathrm{E}+00$ \\
\hline 59.5194 & ${ }^{2} \mathrm{~F}_{5 / 2}^{\circ} 2$ & $\mathrm{M} 1+\mathrm{E} 2$ & $1.25 \mathrm{E}+00$ & $.72 \mathrm{E}+00$ \\
\hline 63 & & E2 & $2.92 \mathrm{E}-01$ & $1.01 \mathrm{E}-01$ \\
\hline 1 & ${ }^{4} \mathbf{I}_{9}^{\circ}$ & $\mathrm{M} 1+\mathrm{E} 2$ & $4.20 \mathrm{E}-01$ & $4.36 \mathrm{E}-01$ \\
\hline 6 & & E2 & $2.22 \mathrm{E}-01$ & $1.01 \mathrm{E}-01$ \\
\hline 68.3601 & ${ }^{2} \mathbf{I}_{13}^{\circ}$ & E2 & $6.97 \mathrm{E}-01$ & \\
\hline 68.4777 & ${ }^{4} \mathrm{I}_{13 / 2}^{\circ}-{ }^{2} \mathrm{G}_{9 / 2}^{\circ} 2$ & E2 & $1.16 \mathrm{E}-01$ & $6.71 \mathrm{E}-02$ \\
\hline 569 & ${ }^{4} \mathrm{~S}_{3}^{\circ}$ & E2 & $E-01$ & $\mathrm{E}-01$ \\
\hline 69 & ${ }^{2} \mathrm{G}_{7 / 2}^{\circ} 2-{ }^{2} \mathrm{D}_{5 / 2}^{\circ} 2$ & $\mathrm{M} 1+\mathrm{E} 2$ & 1.5 & $.35 \mathrm{E}+00$ \\
\hline 8 & ${ }^{4} \mathrm{G}_{5 / 2}^{\circ}-{ }^{2} \mathrm{D}_{5 / 2}^{\circ} 2$ & M1 & $2.48 \mathrm{E}+00$ & $2.30 \mathrm{E}+00$ \\
\hline & ${ }^{4} \mathrm{~F}_{3}^{\circ}$ & $\mathrm{M} 1+\mathrm{E} 2$ & $4.53 \mathrm{E}-01$ & $7.06 \mathrm{E}-01$ \\
\hline 4 & ${ }^{2} \mathrm{H}_{9,}^{\circ}$ & $\mathrm{M} 1+\mathrm{E} 2$ & $1.11 \mathrm{E}+00$ & $1.74 \mathrm{E}+00$ \\
\hline 7 & ${ }^{2} \mathrm{~F}_{7 / 2}^{\circ} 2$ & $\mathrm{M} 1+\mathrm{E} 2$ & $7.32 \mathrm{E}-01$ & \\
\hline 08.8 & ${ }^{2} \mathrm{G}_{7 / 2}^{\circ} 1$ & $\mathrm{M} 1+\mathrm{E} 2$ & $4.26 \mathrm{E}+00$ & \\
\hline 14 & ${ }^{4} \mathrm{~F}_{9 / 2}^{\circ}$ & $\mathrm{M} 1+\mathrm{E} 2$ & $1.70 \mathrm{E}-01$ & $1.67 \mathrm{E}-01$ \\
\hline & ${ }^{2} \mathrm{G}_{7 / 2}^{\circ}$ & $\mathrm{M} 1+\mathrm{E} 2$ & $4.92 \mathrm{E}-01$ & $4.33 \mathrm{E}-01$ \\
\hline & ${ }^{4} \mathbf{I}_{13 / 2}^{\circ}$ & E2 & $E-01$ & $12 \mathrm{E}-02$ \\
\hline ? & ${ }^{4} \mathbf{I}_{15 / 2}^{\circ}$ & E2 & $3.14 \mathrm{E}-01$ & $2.18 \mathrm{E}-01$ \\
\hline & ${ }^{4} \mathrm{~S}_{3 /}^{\circ}$ & $\mathrm{M} 1+\mathrm{E} 2$ & $1.01 \mathrm{E}-01$ & $1.01 \mathrm{E}-01$ \\
\hline & ${ }^{4} \mathrm{G}_{7 / 2}^{\circ}$ & M1 & +00 & $1 \mathrm{E}+00$ \\
\hline & ${ }^{4} \mathrm{~F}_{7 / 2}^{\circ}-{ }^{4} \mathrm{D}_{5 / 2}^{\circ}$ & $\mathrm{M} 1+\mathrm{E} 2$ & $1.93 \mathrm{E}-01$ & $6.26 \mathrm{E}-02$ \\
\hline & ${ }^{2} \mathrm{H}_{11 / 2}^{\circ}$ & $\mathrm{M} 1+\mathrm{E} 2$ & $1.40 \mathrm{E}+00$ & $1.22 \mathrm{E}+00$ \\
\hline 79 & ${ }^{4} S_{3 / 2}^{\circ}$ & $\mathrm{M} 1+\mathrm{E} 2$ & $2.56 \mathrm{E}-01$ & $2.55 \mathrm{E}-01$ \\
\hline & ${ }^{2} \mathrm{D}_{5 / 2}^{\circ}$ & $\mathrm{M} 1+\mathrm{E} 2$ & $3.51 \mathrm{E}-01$ & $1.84 \mathrm{E}-01$ \\
\hline 77 & ${ }^{4} \mathrm{~S}_{3 / 2}^{\circ}-{ }^{4} \mathrm{D}_{3 / 2}^{\circ}$ & $\mathrm{M} 1+\mathrm{E} 2$ & $2.56 \mathrm{E}-01$ & $2.64 \mathrm{E}-01$ \\
\hline 59. & ${ }^{4} \mathrm{G}_{9 / 2}^{\circ}-{ }^{2} \mathrm{H}_{11 / 2}^{\circ} 1$ & $\mathrm{M} 1+\mathrm{E} 2$ & $9.34 \mathrm{E}+00$ & $9.57 \mathrm{E}+00$ \\
\hline 53 & ${ }^{2} \mathrm{~K}_{13 / 2}^{\circ}-{ }^{2} \mathrm{H}_{11 / 2}^{\circ} 1$ & $\mathrm{M} 1+\mathrm{E} 2$ & $9.45 \mathrm{E}-01$ & $9.21 \mathrm{E}-01$ \\
\hline 83 & ${ }^{4} \mathrm{~F}_{3 / 2}^{\circ}-{ }^{2} \mathrm{P}_{3 / 2}^{\circ}$ & M1 & $1.44 \mathrm{E}+00$ & $1.29 \mathrm{E}+00$ \\
\hline 58.8446 & ${ }^{2} \mathrm{D}_{5 / 2}^{\circ} 2-{ }^{2} \mathrm{G}_{7 / 2}^{\circ} 1$ & M1 & $1.71 \mathrm{E}-01$ & \\
\hline 0120 & ${ }^{4} \mathrm{G}_{7 / 2}^{\circ}-{ }^{\circ} \mathrm{H}_{9 / 2}^{\circ} 1$ & $\mathrm{M} 1+\mathrm{E} 2$ & $5.84 \mathrm{E}+00$ & $5.37 \mathrm{E}+00$ \\
\hline & ${ }^{2} \mathrm{H}_{11 / 2}^{\circ} 1-{ }^{2} \mathrm{G}_{9 / 2}^{\circ}$ & $\mathrm{M} 1+\mathrm{E} 2$ & $2.46 \mathrm{E}+00$ & \\
\hline 713.4137 & ${ }^{4} \mathrm{~F}_{5 / 2}^{\circ}-{ }^{\circ} \mathrm{P}_{3 / 2}^{\circ}$ & M1 & $3.62 \mathrm{E}+00$ & $3.43 \mathrm{E}+00$ \\
\hline 8171 & ${ }^{2} \mathrm{~K}_{13 / 2}^{\circ}-{ }^{2} \mathrm{H}_{9 / 2}^{\circ} 1$ & E2 & $1.08 \mathrm{E}-01$ & $5.74 \mathrm{E}-02$ \\
\hline 739.82 & ${ }^{2} \mathrm{D}_{3 / 2}^{\circ} 1-^{2} \mathrm{D}_{5 / 2}^{\circ} 2$ & $\mathrm{M} 1+\mathrm{E} 2$ & $2.40 \mathrm{E}-01$ & $2.63 \mathrm{E}-01$ \\
\hline 763.2792 & ${ }^{4} \mathrm{I}_{11 / 2}^{\circ}-{ }^{\circ} \mathrm{F}_{9 / 2}^{\circ}$ & M1 & $2.74 \mathrm{E}+00$ & $2.51 \mathrm{E}+00$ \\
\hline 770.8751 & ${ }^{4} S_{3 / 2}^{\circ}-{ }^{2} P_{3 / 2}^{o}$ & M1 & $3.09 \mathrm{E}+00$ & $3.15 \mathrm{E}+00$ \\
\hline 780.5942 & ${ }^{2} \mathrm{P}_{3 / 2}^{\circ}-{ }^{\circ} \mathrm{F}_{5 / 2}^{\circ} 2$ & $\mathrm{M} 1+\mathrm{E} 2$ & $1.25 \mathrm{E}+00$ & $1.31 \mathrm{E}+00$ \\
\hline & ${ }^{4} \mathrm{~F}_{3 / 2}^{\circ}-{ }^{2} \mathrm{D}_{5 / 2}^{\circ} 1$ & M1 & $5.73 \mathrm{E}-01$ & $5.06 \mathrm{E}-01$ \\
\hline 815.8291 & ${ }^{4} \mathrm{I}_{13 / 2}^{\circ}-{ }^{2} \mathrm{H}_{11 / 2}^{\circ} 2$ & M1 & $1.23 \mathrm{E}+01$ & $1.11 \mathrm{E}+01$ \\
\hline
\end{tabular}

Table 5. (Continued.)

\begin{tabular}{|c|c|c|c|c|}
\hline $\begin{array}{l}\text { Wavelength }{ }^{\mathrm{a}} \\
(\mathrm{nm})\end{array}$ & Transition & Type $^{\text {b }}$ & $\begin{array}{l}g A \\
\text { (This work) } \\
\left(\mathrm{s}^{-1}\right)\end{array}$ & $\begin{array}{l}g A \\
(\text { Other })^{\mathrm{c}} \\
\left(\mathrm{s}^{-1}\right)\end{array}$ \\
\hline 826.8725 & 1 & M1 & $2.23 \mathrm{E}-01$ & $2.46 \mathrm{E}-01$ \\
\hline 846.2036 & ${ }^{4} \mathrm{G}_{7 / 2}^{\circ}-{ }^{4} \mathrm{D}_{7 / 2}^{\circ}$ & M1 & $1.65 \mathrm{E}-01$ & $1.62 \mathrm{E}-01$ \\
\hline 866.6286 & ${ }^{2} \mathrm{G}_{7 / 2}^{\circ} 2-{ }^{4} \mathrm{D}_{5 / 2}^{\circ}$ & $\mathrm{M} 1+\mathrm{E} 2$ & $1.22 \mathrm{E}-01$ & $1.18 \mathrm{E}-01$ \\
\hline 870.5573 & ${ }^{4} \mathrm{G}_{5 / 2}^{\circ}-{ }^{4} \mathrm{D}_{5 / 2}^{\circ}$ & $\mathrm{M} 1+\mathrm{E} 2$ & $4.94 \mathrm{E}-01$ & $4.69 \mathrm{E}-01$ \\
\hline 878.0883 & ${ }^{4} \mathrm{G}_{9 / 2}^{\mathrm{o} / 2}-{ }^{4} \mathrm{D}_{7 / 2}^{\mathrm{o} / 2}$ & $\mathrm{M} 1+\mathrm{E} 2$ & $1.07 \mathrm{E}-01$ & $9.90 \mathrm{E}-02$ \\
\hline 906.3054 & ${ }^{2} \mathrm{~K}_{13 / 2}^{\circ}-{ }^{2} \mathrm{~L}_{15 / 2}^{\circ}$ & M1 & $1.09 \mathrm{E}+01$ & $1.08 \mathrm{E}+01$ \\
\hline 916.9120 & ${ }^{4} \mathbf{I}_{11 / 2}^{\circ}-{ }^{2} \mathrm{H}_{9 / 2}^{\circ} 2$ & M1 & $7.72 \mathrm{E}+00$ & $7.61 \mathrm{E}+00$ \\
\hline 941.9574 & ${ }^{4} \mathrm{~F}_{7 / 2}^{\circ}-{ }^{\circ} \mathrm{D}_{5 / 2}^{\circ} 1$ & M1 & $8.00 \mathrm{E}+00$ & $7.80 \mathrm{E}+00$ \\
\hline 943.8026 & ${ }^{2} \mathrm{~F}_{5 / 2}^{\circ} 2-{ }^{2} \mathrm{G}_{7 / 2}^{\circ} 1$ & $\mathrm{M} 1+\mathrm{E} 2$ & $3.16 \mathrm{E}-01$ & \\
\hline 946.8902 & ${ }^{4} \mathrm{D}_{3 / 2}^{\circ}-{ }^{2} \mathrm{~F}_{5 / 2}^{\circ} 2$ & $\mathrm{M} 1+\mathrm{E} 2$ & $3.38 \mathrm{E}-01$ & $2.73 \mathrm{E}-01$ \\
\hline 948.4515 & ${ }^{4} S_{3 / 2}^{3 / 2}-{ }^{2} D_{5 / 2}^{5 / 2} 1$ & M1 & $2.81 \mathrm{E}-01$ & $2.89 \mathrm{E}-01$ \\
\hline 950.3277 & ${ }^{2} \mathrm{~K}_{15 / 2}^{\circ}-{ }^{2} \mathrm{~L}_{17 / 2}^{\circ}$ & M1 & $1.08 \mathrm{E}+01$ & $1.06 \mathrm{E}+01$ \\
\hline 953.3655 & ${ }^{2} \mathrm{P}_{1 / 2}^{\circ}-{ }^{\circ} \mathrm{D}_{3 / 2}^{\circ} 2$ & $\mathrm{M} 1+\mathrm{E} 2$ & $5.65 \mathrm{E}-01$ & $4.54 \mathrm{E}-01$ \\
\hline 979.1500 & ${ }^{4} \mathrm{G}_{9 / 2}^{\mathrm{o}}-{ }^{2} \mathrm{I}_{11 / 2}^{\mathrm{o} / 2}$ & M1 & $7.16 \mathrm{E}-01$ & $6.00 \mathrm{E}-01$ \\
\hline 982.5596 & ${ }^{2} \mathrm{~K}_{13 / 2}^{\circ}-{ }^{2} \mathrm{I}_{11 / 2}^{o}$ & $\mathrm{M} 1+\mathrm{E} 2$ & $1.50 \mathrm{E}+00$ & $1.48 \mathrm{E}+00$ \\
\hline 999.4850 & ${ }^{4} \mathrm{~F}_{3 / 2}^{\circ}-{ }^{2} \mathrm{D}_{3 / 2}^{\circ} 1$ & M1 & $2.89 \mathrm{E}+00$ & $2.85 \mathrm{E}+00$ \\
\hline 1000.0819 & ${ }^{4} \mathrm{~S}_{3 / 2}^{\circ}-{ }^{2} \mathrm{P}_{1 / 2}^{\mathrm{o}}$ & M1 & $1.69 \mathrm{E}+00$ & $1.64 \mathrm{E}+00$ \\
\hline 1005.5470 & ${ }^{2} \mathrm{D}_{5 / 2}^{\circ} 1-^{2} \mathrm{D}_{3 / 2}^{\circ} 2$ & M1 & $3.57 \mathrm{E}+00$ & $3.73 \mathrm{E}+00$ \\
\hline 1048.0319 & ${ }^{2} \mathrm{~K}_{15 / 2}^{\mathrm{o} / 2}$ & M1 & $1.48 \mathrm{E}+00$ & $1.17 \mathrm{E}+00$ \\
\hline 1116.6432 & ${ }^{4} \mathrm{~F}_{5 / 2}^{\circ}-{ }^{2} \mathrm{D}_{3 / 2}^{\circ} 1$ & M1 & $3.64 \mathrm{E}+00$ & $3.69 \mathrm{E}+00$ \\
\hline 1182.7260 & ${ }^{2} \mathrm{P}_{3 / 2}^{\circ}-{ }^{2} \mathrm{D}_{5 / 2}^{\circ} 2$ & M1 & $2.37 \mathrm{E}+00$ & $1.97 \mathrm{E}+00$ \\
\hline 1217.1925 & ${ }^{4} \mathrm{D}_{7 / 2}^{\circ}-{ }^{\circ} \mathrm{F}_{5 / 2}^{\circ / 2} 2$ & M1 & $1.87 \mathrm{E}+00$ & $1.57 \mathrm{E}+00$ \\
\hline 1225.1925 & ${ }^{2} \mathrm{~F}_{7 / 2}^{\circ} 2-{ }^{2} \mathrm{G}_{9 / 2}^{\circ} 1$ & $\mathrm{M} 1+\mathrm{E} 2$ & $2.30 \mathrm{E}-01$ & \\
\hline 1264.1257 & ${ }^{4} \mathrm{~S}_{3 / 2}^{\circ}-{ }^{\circ} \mathrm{D}_{3 / 2}^{\circ} 1$ & M1 & $4.55 \mathrm{E}+00$ & $4.32 \mathrm{E}+00$ \\
\hline 1275.2411 & ${ }^{2} \mathrm{D}_{3 / 2}^{\circ} 1-{ }^{4} \mathrm{D}_{1 / 2}^{\circ}$ & M1 & $1.97 \mathrm{E}-01$ & $2.44 \mathrm{E}-01$ \\
\hline 1286.0584 & ${ }^{4} \mathrm{~F}_{7 / 2}^{\circ / 2}-{ }^{2} \mathrm{G}_{9 / 2}^{\circ} 2$ & M1 & $2.93 \mathrm{E}+00$ & $2.52 \mathrm{E}+00$ \\
\hline 1334.7467 & ${ }^{2} \mathrm{D}_{3 / 2}^{\circ} 1-{ }^{4} \mathrm{D}_{5 / 2}^{\circ}$ & M1 & $2.10 \mathrm{E}+00$ & $1.91 \mathrm{E}+00$ \\
\hline 1417.5454 & ${ }^{4} \mathrm{~F}_{9 / 2}^{\circ}-{ }^{4} \mathrm{G}_{11 / 2}^{\circ}$ & $\mathrm{M} 1+\mathrm{E} 2$ & $1.46 \mathrm{E}-01$ & $3.21 \mathrm{E}-01$ \\
\hline 1471.7317 & ${ }^{4} \mathrm{~F}_{5 / 2}^{\circ}-{ }^{4} \mathrm{G}_{7 / 2}^{\circ}$ & M1 & $1.34 \mathrm{E}+00$ & $1.06 \mathrm{E}+00$ \\
\hline 1497.0474 & ${ }^{2} \mathrm{G}_{7 / 2}^{\circ} 2-{ }^{2} \mathrm{D}_{5 / 2}^{\circ} 1$ & M1 & $1.37 \mathrm{E}-01$ & $1.86 \mathrm{E}-01$ \\
\hline 1538.3915 & ${ }^{4} \mathrm{~F}_{9 / 2}^{\circ}-{ }^{2} \mathrm{G}_{9 / 2}^{\circ} 2$ & M1 & $9.19 \mathrm{E}+00$ & $7.80 \mathrm{E}+00$ \\
\hline 1599.5707 & ${ }^{4} \mathrm{~F}_{7 / 2}^{\circ}-{ }^{4} \mathrm{G}_{9 / 2}^{\circ}$ & M1 & $4.23 \mathrm{E}-01$ & $4.77 \mathrm{E}-01$ \\
\hline 1611.5555 & ${ }^{4} \mathrm{D}_{3 / 2}^{\circ}-{ }^{2} \mathrm{D}_{5 / 2}^{\circ} 2$ & M1 & $3.25 \mathrm{E}+00$ & $3.10 \mathrm{E}+00$ \\
\hline 1663.8045 & ${ }^{4} \mathrm{~F}_{3 / 2}^{\circ / 2}-{ }^{4} \mathrm{G}_{5 / 2}^{\circ}$ & $\mathrm{M} 1+\mathrm{E} 2$ & $3.14 \mathrm{E}-02$ & \\
\hline 1717.4547 & ${ }^{4} \mathrm{~F}_{7 / 2}^{\circ}-{ }^{4} \mathrm{G}_{7 / 2}^{\circ}$ & M1 & $1.34 \mathrm{E}+00$ & \\
\hline 1724.0298 & ${ }^{2} \mathrm{D}_{5 / 2}^{\circ} 2-^{2} \mathrm{~F}_{7 / 2}^{\circ} 2$ & M1 & $4.37 \mathrm{E}+00$ & \\
\hline 1738.2082 & ${ }^{2} \mathrm{P}_{1 / 2}^{\circ}-{ }^{\circ} \mathrm{D}_{1 / 2}^{\circ}$ & M1 & $2.12 \mathrm{E}-01$ & \\
\hline 1875.0061 & ${ }^{2} \mathrm{H}_{11 / 2}^{\circ} 2{ }^{\circ} \mathrm{G}_{9 / 2}^{\circ} 2$ & M1 & $3.23 \mathrm{E}+00$ & \\
\hline 1888.6971 & ${ }^{2} \mathrm{D}_{3 / 2}^{\circ} 2-{ }^{2} \mathrm{~F}_{5 / 2}^{\circ} 2$ & M1 & $1.89 \mathrm{E}+00$ & \\
\hline 1898.8961 & ${ }^{4} \mathrm{D}_{3 / 2}^{\circ}-{ }^{2} \mathrm{D}_{3 / 2}^{\circ} 2$ & M1 & $1.27 \mathrm{E}+00$ & \\
\hline 1914.6357 & ${ }^{2} \mathrm{P}_{1 / 2}^{\circ / 2}-{ }^{4} \mathrm{D}_{3 / 2}^{\circ}$ & $\mathrm{M} 1+\mathrm{E} 2$ & $6.89 \mathrm{E}-01$ & \\
\hline 1966.3023 & ${ }^{4} \mathrm{D}_{5 / 2}^{\circ}-{ }^{2} \mathrm{D}_{3 / 2}^{\circ}{ }^{\circ} 2$ & M1 & $6.37 \mathrm{E}-01$ & \\
\hline 1975.6320 & ${ }^{2} \mathrm{D}_{3 / 2}^{\circ} 1-{ }^{2} \mathrm{P}_{3 / 2}^{\circ}$ & M1 & $4.28 \mathrm{E}+00$ & \\
\hline
\end{tabular}

${ }^{\text {a }}$ Vacuum (below $200 \mathrm{~nm}$ ) and air (above $200 \mathrm{~nm}$ ) wavelengths deduced from the experimental energy level values of Wyart et al (2007).

${ }^{\mathrm{b}}$ Contributions larger than $1 \%$.

${ }^{\mathrm{c}}$ From Dodson and Zia (2012).

Another comparison can be made for radiative lifetimes which were also reported for some $4 \mathrm{f}^{2} 5 \mathrm{~d}$ levels by Wyart et al (2007) and by Dzuba et al (2003). Such a comparison is given in table 4 . As seen from this table, while a satisfactory agreement (within 20\%) is found between our results and those of Wyart et al (2007), larger discrepancies (generally reaching a factor of 2 or even more) are observed when comparing our computed lifetimes with those of Dzuba et al (2003). However, 


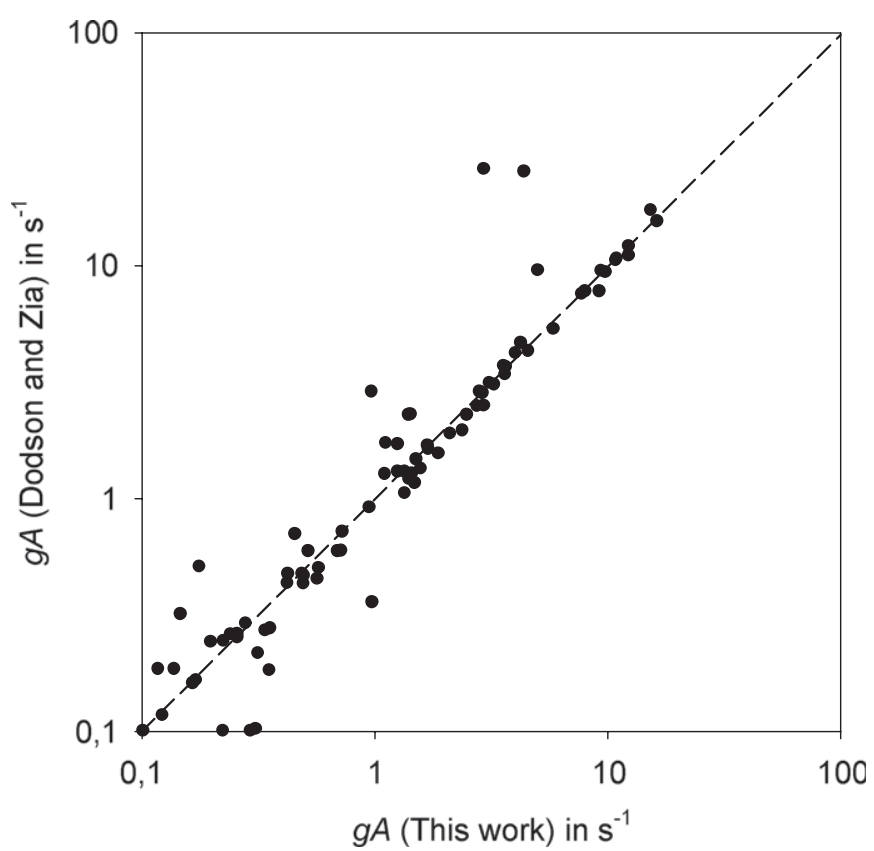

Figure 3. Comparison between calculated radiative transition probabilities as obtained in the present work and those published by Dodson and Zia (2012) for forbidden lines in Nd IV.

it is worth mentioning that these latter authors did not include core-polarization and correlation effects in their calculations and, as a consequence, estimated their computed lifetimes to be too small by a factor of 3 or 4 .

\subsection{Forbidden transitions}

Triply ionized lanthanides are known to exhibit very characteristic emission lines in the visible and near infrared regions due to $4 \mathrm{f} \rightarrow 4 \mathrm{f}$ transitions. These transitions, forbidden by the electric dipole selection rules, are characterized by long lifetimes which facilitate 'time gated' emission experiments leading to significant improvement of signal-to-noise ratios compared with more traditional steady-state measurements. For this reason, radiative properties of such forbidden lines within the $4 \mathrm{f}^{3}$ configurations were also computed in the present work. Transition probabilities for the most intense magnetic dipole (M1) and electric quadrupole (E2) lines are reported in table 5 where they are compared to the data recently published by Dodson and Zia (2012). These latter authors reported radiative transition probabilities for some M1 and E2 Nd IV lines using a detailed free ion Hamiltonian including electrostatic and spin-orbit terms as well as two-body, three-body, spin-spin, spin-other-orbit and electrostatically correlated spin-orbit interactions. As seen from table 5, for M1 lines, our $g A$-values are generally in good agreement (within a few per cent) with those of Dodson and $\mathrm{Zia}$ while larger discrepancies (reaching 30\%-40\%) can be observed for some E2 lines. Nevertheless, as illustrated in figure 3 showing the overall comparison between both sets of transition probabilities, it is clear that a satisfactory agreement is observed for the most intense forbidden lines, i.e. for those characterized by $g A$-values larger than $1 \mathrm{~s}^{-1}$.

\section{Conclusion}

A new set of radiative parameters has been obtained for electric dipole, magnetic dipole and electric quadrupole lines in triply ionized neodymium using a pseudo-relativistic Hartree-Fock model combined with a semi-empirical optimization of electrostatic and spin-orbit radial integrals. Computed transition probabilities, oscillator strengths and radiative lifetimes have been compared with other available theoretical data. For electric dipole transitions, our results are expected to be more accurate than those previously published in view of the much more extended amount of electronic correlation effects (including core-excited correlation) considered in our physical model. In the case of magnetic dipole and electric quadrupole lines, our results have been found to be in good agreement with theoretical data recently published for the most intense transitions.

\section{Acknowledgments}

The authors acknowledge financial support from the Belgian National Fund for Scientific Research (F.R.S.-FNRS) from which PQ is a Research Director. SEY also thanks the Marien Ngouabi University for its support.

\section{References}

Biémont É, Clar M, Enzonga Yoca S, Fivet V, Quinet P, Träbert E and Garnir H-P 2009 Can. J. Phys. 871275

Biémont É, Garnir H-P, Palmeri P, Quinet P, Li Z S, Zhang Z G and Svanberg S 2001b Phys. Rev. A 64022503

Biémont É and Quinet P 2003 Phys. Scr. T 10538

Cowan R D 1981 The Theory of Atomic Structure and Spectra (Berkeley, CA: University of California Press)

Crosswhite H M, Crosswhite H, Kaseta F W and Sarup R 1976 J. Chem. Phys. 641981

Dodson C M and Zia R 2012 Phys. Rev. B 86125102

Dossing A 2005 Eur. J. Inorg. Chem. 20051425

Dzuba V A, Safronova U I and Johnson W R 2003 Phys. Rev. A 68032503

Enzonga Yoca S and Quinet P 2013 J. Phys. B: At. Mol. Opt. Phys. 46145003

Gasparik V and Ozvoldova M 1974 J. Phys. B: At. Mol. Phys. 24699

Hasegawa Y, Wada Y and Yanagida S 2004 J. Photochem. Photobiol. C 5183

Hemmila I 1995 J. Alloys Compounds 225480

Irwin D J G 1968 PhD Thesis (Baltimore, MD: Johns Hopkins University)

Martin W C, Zalubas R and Hagan L 1978 Atomic Energy Levels The Rare Earth Elements NSRDS-NBS 60 (Washington, DC: US Government Printing Office)

Palmeri P, Quinet P, Wyart J-F and Biémont É 2000a Phys. Scr. 61323

Palmeri P, Quinet P, Frémat Y, Wyart J-F and Biémont É 2000b Astrophys. J. Suppl. Ser. 129367

Quinet P, Palmeri P, Biémont, Li Z S, Zhang Z G and Svanberg S 2002 J. Alloys Compounds $\mathbf{3 4 4} 255$

Rao J 1973 Indian J. Pure Appl. Phys. 11833

Spector N, Guttel C and Reisfeld R 1977 Opt. Pura Apl. 10197

Vaishnava P P, Tandon S P and Bhutra M P 1974 Spectrosc. Lett. 7515

Wortman D E, Karayianis N and Morrison C A 1974 Harry Diamond Laboratories Report HDL-TR-1685 (Washington DC: HDL) 
Wyart J-F, Tchang-Brillet W-Ü L, Spector N, Palmeri P, Quinet P and Biémont É 2001 Phys. Scr. 63113

Wyart J-F, Meftah A, Bachelier A, Sinzelle J,

Tchang-Brillet W-Ü L, Champion N, Spector N and Sugar J 2006 J. Phys. B: At. Mol. Opt. Phys. 39 L77

Wyart J-F, Meftah A, Tchang-Brillet W-Ü L, Champion N, Lamrous O, Spector N and Sugar J 2007 J. Phys. B: At. Mol. Opt. Phys. 403957
Wyart J-F, Meftah A, Sinzelle J, Tchang-Brillet W-Ü L, Spector N and Judd B R 2008 J. Phys. B: At. Mol. Opt. Phys. 41085001 Wybourne B G 2004 J. Alloys Compounds 38096

Zalucha D J, Sell J A and Fong F K 1974 J. Chem. Phys. 601660

Zhang Z G, Svanberg S, Quinet P, Palmeri P and Biémont É 2001a Phys. Rev. Lett. 87273001

Zhang Z G, Svanberg S, Jiang Z, Palmeri P, Quinet P and Biémont É 2001b Phys. Scr. 63122 